\title{
A hierarchy of models for type-II superconductors
}

\author{
S. J. Chapman \\ Mathematical Institute, \\ 24-29 St. Giles, \\ Oxford OX1 3LB
}

March 26, 2004

\begin{abstract}
A hierarchy of models for type-II superconductors is presented. Through appropriate asymptotic limits we pass from the mesoscopic Ginzburg-Landau model to the London model with isolated superconducting vortices as line singularities, to vortex-density models, and finally to macroscopic critical-state models.
\end{abstract}

Keywords. superconductivity, vortices, homogenisation, critical state, Bean, vortex density, London, Ginzburg-Landau.

\section{Introduction}

Since the discovery of high-temperature superconducting materials in 1986 there has been a huge resurgence in interest in superconductivity in both the engineering and physics communities, not only at microscopic level, but also at the meso- and macroscopic levels. During this time there has also been an enormous increase in activity in the subject by mathematicians.

The explosion of work in the engineering community is largely a result of the fact that because high-temperature materials can operate at temperatures within the cooling capabilities of liquid nitrogen it is becoming economically feasible to build more and more devices out of superconducting materials. Traditional low-temperature superconducting devices include Nuclear Magnetic Resonance (NMR) machines, in which the superconductor is used to produce a large magnetic field with no energy loss, and Superconducting Quantum Interference Devices (SQUIDs), which are instruments which measure a magnetic field very accurately, and which utilise the macroscopic quantum properties of superconductivity.

With the advent of high-temperature materials engineers are examining the possibility of using superconductors in other applications, in place of more traditional materials. One example under development is the superconducting motor, which utilises a superconducting magnet in place of the traditional permanent magnet. The simplest configuration, one of a number of prototypes under development in the Department of Engineering at Oxford University ${ }^{1}$, is shown in Figure 1a. There a superconducting puck is magnetically levitated above a copper coil carrying an alternating current. After a short start up period the puck rotates synchronously with the current in the coil (in the actual motor the superconductor would be a longer cylinder and would sit down inside the copper coil; the levitating puck has been shown for clarity).

\footnotetext{
${ }^{1}$ see http://www.eng.ox.ac.uk/ epgmdm
} 


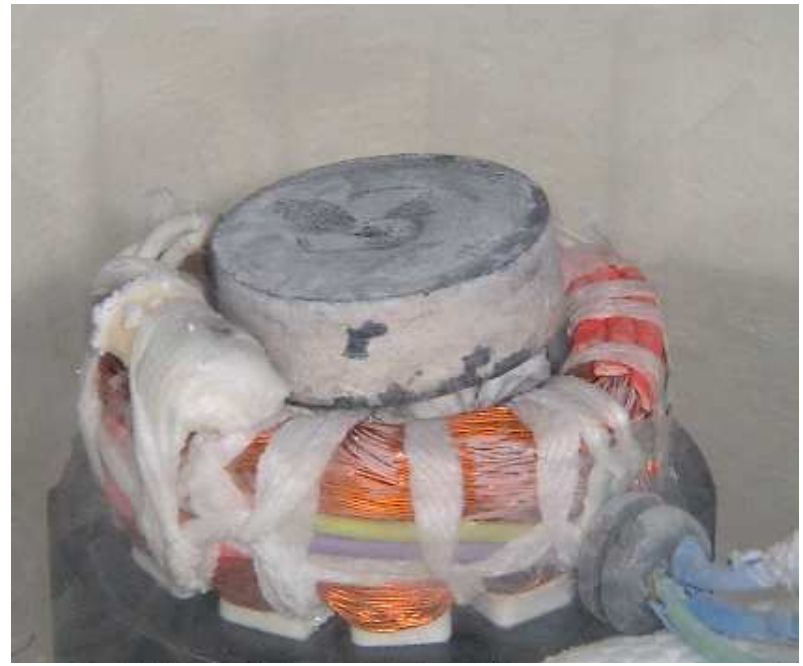

(a)

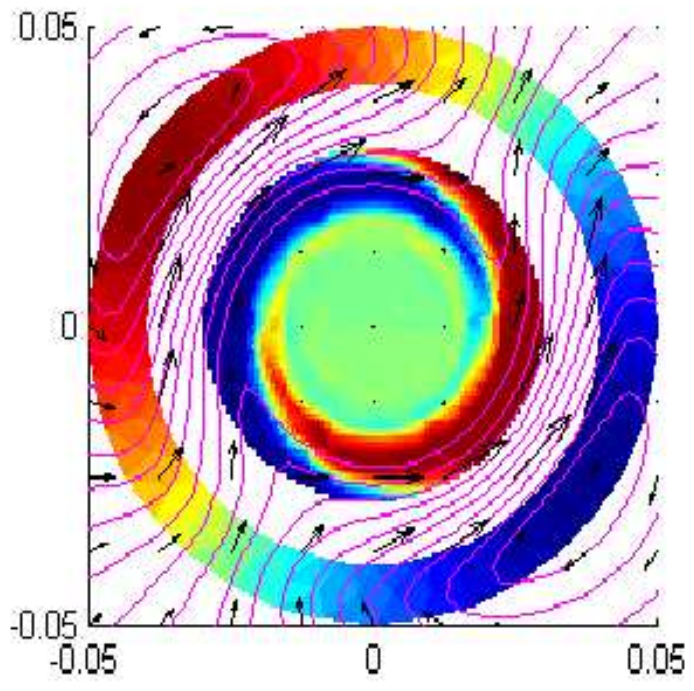

(b)

Figure 1: (a) A photograph of the superconducting motor under development at the Department of Engineering at Oxford University. A superconducting puck levitates above a stator carrying an alternating electric current. After a short start-up period the rotor rotates synchronously with the stator current, (b) An example of the numerical solution of the two-dimensional Bean criticalstate model (equations (205)-(206) coupled with Maxwell's equations). The central cylinder is the superconducting rotor, while the outer annulus is the stator housing an applied rotating sinusoidal current. The colour indicates the current density (blue coming out of the paper, red moving into the paper), while the contour lines indicate the direction of the magnetic field. (Numerical solution due to G. Barnes $[6,5]$.)

An example of the numerical solution of the two-dimensional Bean critical-state model (equations (205)-(206) coupled with Maxwell's equations) describing the magnetic field and electric current in a cross-section of the superconducting cylinder (assuming the cylinder has a large aspect ratio) is shown in Figure 1b [5]. This can be used to determine the torque on the superconducting rotor, and hence the power of the motor $[6,5]$.

A linear version of this motor has also been designed, and has been used to examine the possibilities of magnetically levitated (maglev) trains. A small experimental maglev "train", developed at the University of the Witwatersrand, Johannesburg in collaboration with the Department of Engineering at Oxford University is shown in Figure 2a. The corresponding numerical solution of the two-dimensional Bean critical-state model in shown in Figure 2b. The rectangles are levitating superconducting components of the "train", while the track is a combination of current carrying wire (the coloured part) and iron armatures (the grey part). The oscillating current in the track provides the magnetic field to levitate the train and drive it forwards.

Japan in particular has quite a serious maglev train project, and indeed the Japanese Railway Technology Research Institute have a $42.8 \mathrm{~km}$ long test track on which a five-car manned train set achieved a maximum speed of $552 \mathrm{~km} / \mathrm{h}$ in April 1999. 


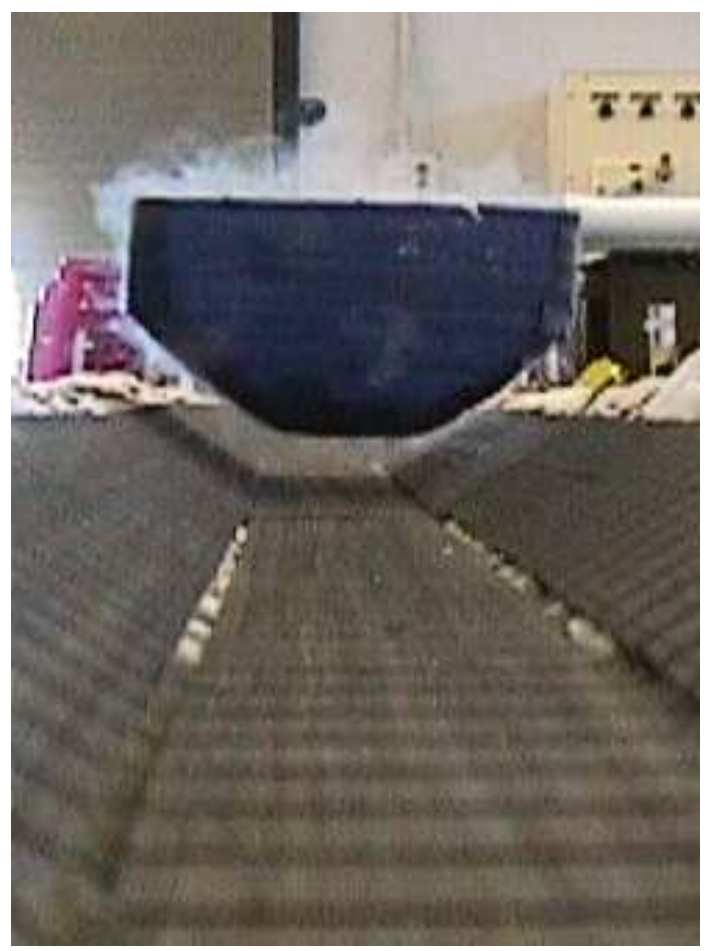

(a)

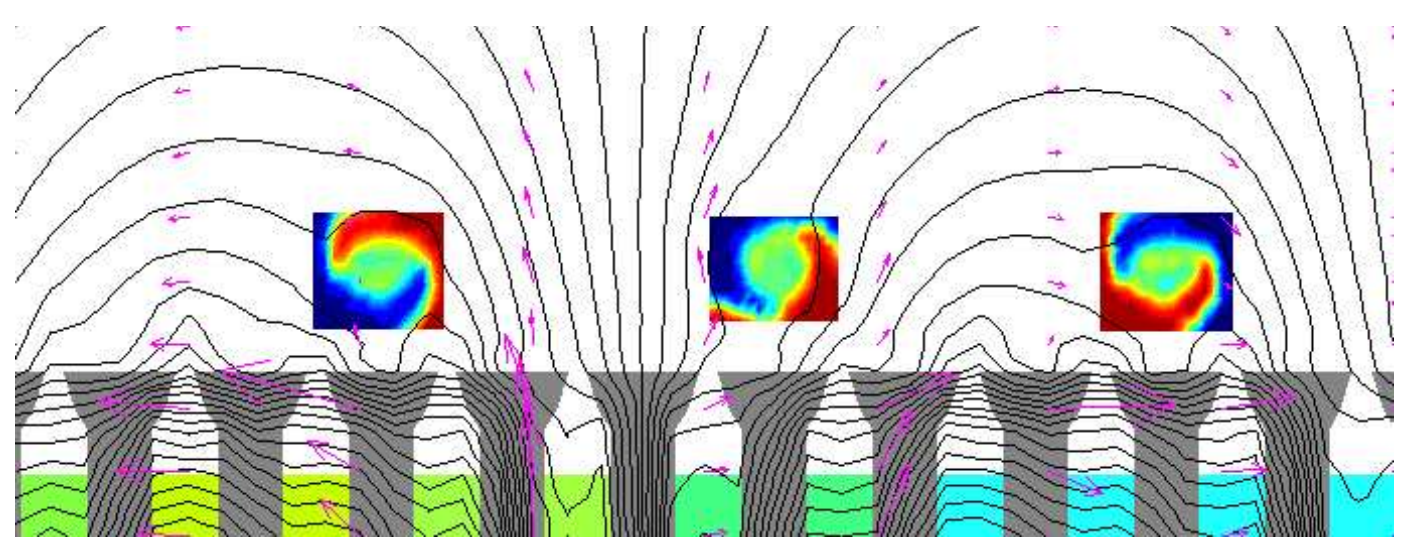

(b)

Figure 2: (a) A photograph of the magnetically levitating "train" under development at the University of the Witwatersrand, Johannesburg in collaboration with the Department of Engineering at Oxford University, (b) An example of the numerical solution of the two-dimensional Bean criticalstate model (equations (205)-(206) coupled with Maxwell's equations). The levitating rectangles are the superconducting components of the "train", while the track is a combination of current carrying wire (the coloured part) and iron armatures (the grey part). The oscillating current in the track provides the magnetic field to levitate the train and drive it forwards. The colour indicates the current density (blue coming out of the paper, red moving into the paper), while the contour lines show the direction of the magnetic field. (Numerical solution due to G. Barnes.) 
The numerical simulations shown in Figures $1 \mathrm{~b}$ and $2 \mathrm{~b}$ are both two-dimensional. In reality the aspect ratio of the superconductor is only about two or three at best, and so end effects will play an important part, requiring the solution of the Bean model in three-dimensions. Unfortunately there is no generally accepted three-dimensional version of this model, for reasons we will describe in Sections 4 and 5, and one of the aims of this paper is to highlight this problem and propose a solution.

Besides these large-scale devices, other smaller-scale devices are also under development. Consider, for example, the other electrical components in the circuit which is driving the motor. If they are outside the cooling jacket then heat will invade the motor through the electrical connections. On the other hand, if they are inside, then they need to work at very low temperatures (the operating temperature, even for high-temperature materials, is about $77 \mathrm{~K}$ ). Thus it is natural to try and design these components also to be made from superconducting materials. One such component, also under development in by the group in Oxford, is the superconducting fault current limiter.

The traditional applications involving superconductors as magnets are typically static. However, in both the superconducting motor and the fault current limiter it is important to know the dynamic response of the material. With superconducting devices being designed over a range of lengthscales, from metres to microns, and to operate over a range of timescales, it is important to have a corresponding range of models to cover all experimental and technological situations. Most importantly, it is crucial to understand the assumptions underlying a particular model, so that its range of validity of application can be assessed. The aim of this paper is to bring together many of the models which are used to describe superconducting materials and to show how they may be related through suitable asymptotic limits.

Before we introduce the first of our models, let us describe some of the basic properties of superconducting materials. The transition from normally conducting (normal) to superconducting is usually associated with a critical temperature $T_{c}$, below which the superconductor is able to pass an electric current without an associated electric field, that is, it exhibits perfect conductivity. Here we will be concerned with the response of a superconducting material to an applied magnetic field under isothermal conditions below its critical temperature. This response is conveniently described by Figure 3, which shows the phase the superconductor adopts as a function of the external magnetic field $H_{\text {ext }}$ and the material parameter $\kappa$ (known as the Ginzburg-Landau parameter) which determines the type of superconducting material; $\kappa<1 / \sqrt{2}$ describes what are known as type-I superconductors, while $\kappa>1 / \sqrt{2}$ describes what are known as type-II superconductors.

For type-I superconductors in sufficiently low magnetic fields the material is in the superconducting state, and the field is excluded from the interior of the sample except in thin boundary layers (this effect is known as the Meissner effect). However, there is a critical magnetic field, $H_{c}$, above which the material will revert to the normally conducting (normal) state, and the magnetic field will penetrate it fully.

In type-II superconductors this critical magnetic field splits into a lower critical field, $H_{c_{1}}$ and an upper critical field $H_{c_{2}}$. For magnetic fields below $H_{c_{1}}$ the material is in the superconducting state and the field is excluded from the interior, while for magnetic fields above $H_{c_{2}}$ the material is in the normal state and the field penetrates it fully. For magnetic fields between $H_{c_{1}}$ and $H_{c_{2}}$ a third state exists, known as the "mixed state", in which there is a partial penetration of the magnetic field into the superconducting material, which occurs by means of thin filaments of non-superconducting material carrying magnetic flux ("flux tubes") and circled by a vortex of superconducting current (hence these filaments are often referred to as vortices).

Most technological applications of superconducting materials involve type-II superconductors in high magnetic fields. Thus we will be concerned with determining the behaviour of superconductors 


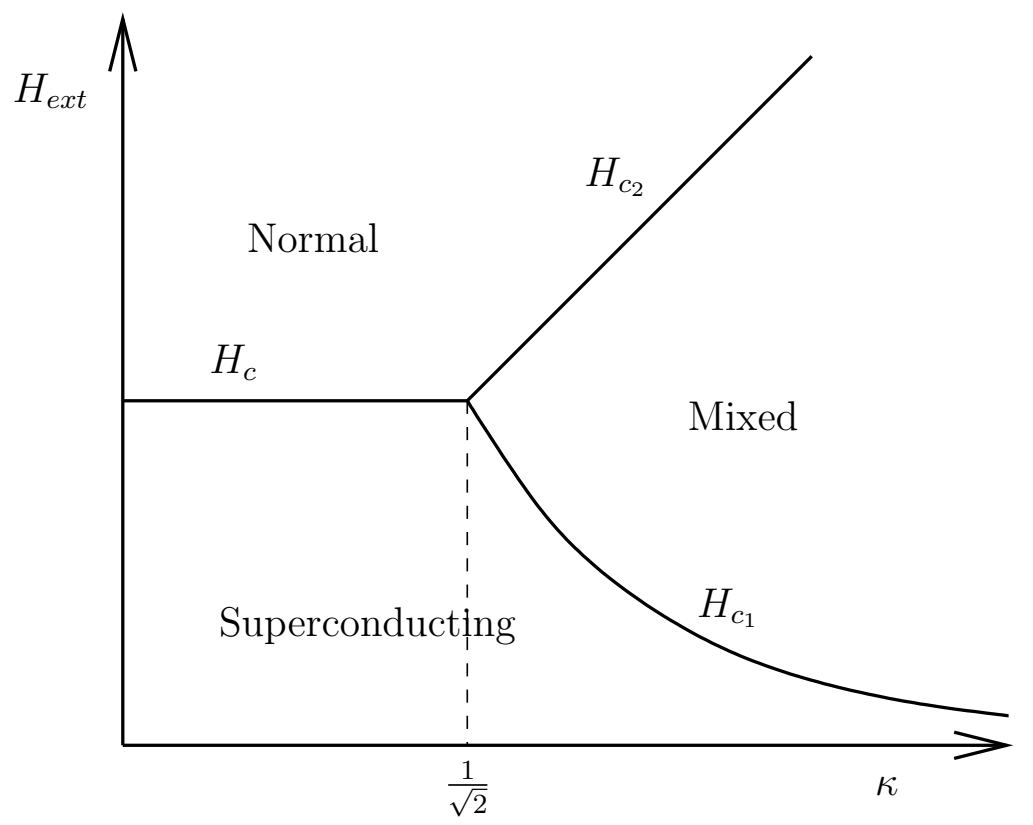

Figure 3: The response of a superconducting material as a function of the applied magnetic field $H_{\text {ext }}$ and the Ginzburg-Landau parameter $\kappa$.

while they are in the mixed state.

The superconducting vortices of the mixed state are well described by the mesoscopic GinzburgLandau model of superconductivity, which is popular among mathematicians and physicists, and which comprises coupled equations for the density of superconducting electrons and the local magnetic field (or more properly the magnetic vector potential). However, the cores of these filaments are typically very small $(\sim 0.1 \mu \mathrm{m})$ and so there are many millions in any real superconducting sample. In designing magnets and other large scale applications of superconducting materials, engineers use more macroscopic models (such as the aforementioned Bean model), which involve bulk variables such as the local average of the magnetic field and electric current, and in which the behaviour of individual vortices is not so important. The superconducting properties of the material appear in these models through a modification of Ohm's law for normally conducting materials; usually they are so-called critical-state models.

We will relate the mesoscopic Ginzburg-Landau theory to the macroscopic critical-state theories through a series of asymptotic limits which will generate a hierarchy of models. We begin in Section 2 by describing the Ginzburg-Landau model and its modification to include pinning effects. In Section 3 we describe a limit of the Ginzburg-Landau model which is known as the London model, and in which vortices appear as line singularities. In Sections 4 and 5 we average over many vortices to produce vortex-density models. In Section 6 we include pinning in these vortex-density models, homogenising the pinning potential to produce critical-state models. Finally, in Section 7 , we present our conclusions.

Where possible, comments are made about the theoretical knowledge of the model being presented. However, many of the models have received as yet little attention in the mathematical literature. 


\section{The Ginzburg-Landau Equations}

The starting point for our discussion of models of superconductivity is the Ginzburg-Landau equations. In their 1950 paper Ginzburg \& Landau [36] introduced the complex superconducting order parameter $\Psi$, which is such that $|\Psi|^{2}$ represents the number density of superconducting charge carriers (Cooper pairs). The need for $\Psi$ to be complex is associated with the macroscopic quantum nature of superconductivity; $\Psi$ can be thought of as an averaged macroscopic wavefunction of the superconducting electrons. The ultimate justification for a complex order parameter came in 1957, when Gor'kov [37] demonstrated that the Ginzburg-Landau equations could be derived as a limit of the microscopic theory of Bardeen, Cooper \& Schrieffer (BSC) [4].

Ginzburg \& Landau proceed in accordance with Landau's general theory of second-order phase transitions by expanding the Helmholtz free energy density $\mathcal{F}$ as a power series in $|\Psi|^{2}$ which is truncated after the second term since the theory is supposed to hold in the vicinity of the critical temperature, where $|\Psi|$ is small. Thus, in the absence of a magnetic field

$$
\mathcal{F}_{s 0}=\mathcal{F}_{n 0}+a(T)|\Psi|^{2}+\frac{b(T)}{2}|\Psi|^{4},
$$

where $\mathcal{F}_{s 0}\left(\mathcal{F}_{n 0}\right)$ is the Helmholtz free energy density of the superconducting (normal) phase in the absense of a magnetic field. In a stable equilibrium we require

$$
\frac{\partial \mathcal{F}_{s 0}}{\partial|\Psi|^{2}}=0, \quad \frac{\partial^{2} \mathcal{F}_{s 0}}{\partial\left(|\Psi|^{2}\right)^{2}}>0,
$$

with $|\Psi|^{2}=0$ for $T>T_{c}$ and $|\Psi|^{2}>0$ for $T<T_{c}$. Hence we need

$$
\begin{array}{ll}
a(T)<0 & \text { for } T<T_{c}, \\
a(T)=0 & \text { for } T=T_{c}, \\
a(T)>0 & \text { for } T>T_{c}
\end{array}
$$

while $b\left(T_{c}\right)>0$. Then the free energy density is minimised by $|\Psi|=0$ if $T>T_{c}$ and by $|\Psi|^{2}=$ $\Psi_{0}^{2} \equiv-a / b$ if $T<T_{c}$. To allow for spatial variation in $\Psi$, Ginzburg \& Landau then added a kinetic energy/surface energy term

$$
\frac{1}{2 m_{s}}\left|\hbar \nabla \Psi-i e_{s} \boldsymbol{A} \Psi\right|^{2},
$$

where $\boldsymbol{A}$ is the magnetic vector potential, which is such that the magnetic induction $\boldsymbol{B}$ satisfies

$$
\boldsymbol{B}=\operatorname{curl} \boldsymbol{A},
$$

and which is determined up to a gradient, $\hbar$ is Planck's constant, $e_{s}$ is the charge of the superconducting charge carriers, which is twice the electronic charge $e$, and $m_{s}$ is in fact somewhat arbitrary (since it may be removed by a rescaling of $\Psi$ ) but is usually taken to be twice the electron mass $m$. Finally, to calculate the Helmholtz free energy density $\mathcal{F}_{s H}$ in the presence of a magnetic field we need to add the magnetic field energy density

$$
\frac{\boldsymbol{H} \cdot \boldsymbol{B}}{2},
$$

where $\boldsymbol{H}$ is the magnetic field, which is related to $\boldsymbol{B}$ through the material law $\boldsymbol{B}=\mu \boldsymbol{H}$ where $\mu$ is the permeability which we will assume is a scalar constant. 
The Gibbs free energy differs from the Helmholtz free energy in the presence of an applied magnetic field $\boldsymbol{H}_{\text {ext }}$ due to the work done by the electromotive force induced by the applied field, so that the Gibbs free energy density is given by

$$
\begin{aligned}
\mathcal{G}_{s H} & =\mathcal{F}_{s H}-\boldsymbol{B} \cdot \boldsymbol{H}_{\text {ext }}, \\
& =\mathcal{F}_{n 0}+a(T)|\Psi|^{2}+\frac{b(T)}{2}|\Psi|^{4}+\frac{1}{4 m}|\hbar \nabla \Psi-2 i e \boldsymbol{A} \Psi|^{2}+\frac{\mu}{2} \boldsymbol{H} \cdot\left(\boldsymbol{H}-2 \boldsymbol{H}_{\text {ext }}\right) .
\end{aligned}
$$

In the region exterior to the superconductor only the last term representing the magnetic field energy is present.

The basic postulate of the Ginzburg-Landau theory is that the total Gibbs free energy should be minimised.

In the normal state $\Psi=0$, and the energy is minimised by a fully penetrating magnetic field $\boldsymbol{H}=\boldsymbol{H}_{\text {ext }}$, giving a free energy density $\mathcal{G}_{s H}=\mathcal{F}_{n 0}-\frac{1}{2} \mu H_{0}^{2}$. In the perfect superconducting state, in the absence of surface effects, $|\Psi|^{2}=-a / b, \boldsymbol{A}=\boldsymbol{H}=\mathbf{0}$, and the free energy density $\mathcal{G}_{s H}=\mathcal{F}_{n 0}-\frac{a^{2}}{2 b}$. Thus we see that the thermodynamic critical field is given by

$$
H_{c}=\frac{|a(T)|}{\sqrt{\mu b(T)}} .
$$

If the superconductor occupies a region $\Omega \subset \mathbb{R}^{3}$ the Euler-Lagrange equations associated with the minimisation of the free energy with respect to $\Psi^{*}$ and $\boldsymbol{A}$ are the celebrated Ginzburg-Landau equations

$$
\begin{aligned}
\frac{1}{4 m}(\hbar \nabla-2 i e \boldsymbol{A})^{2} \Psi & =a(T) \Psi+b(T)|\Psi|^{2} \Psi \quad \text { in } \Omega, \\
\frac{1}{\mu}(\operatorname{curl} \boldsymbol{A})^{2} & =-\frac{i e \hbar}{2 m}\left(\Psi^{*} \nabla \Psi-\Psi \nabla \Psi^{*}\right)+\frac{2 e^{2}}{m}|\Psi|^{2} \boldsymbol{A}+\operatorname{curl} \boldsymbol{H}_{\text {ext }} \quad \text { in } \Omega, \\
\frac{1}{\mu}(\operatorname{curl} \boldsymbol{A})^{2} & =\operatorname{curl} \boldsymbol{H}_{\text {ext }} \quad \text { outside } \Omega,
\end{aligned}
$$

with the natural boundary conditions

$$
\begin{aligned}
\boldsymbol{n} \cdot(\hbar \nabla-2 i e \boldsymbol{A}) \Psi & =0 \quad \text { on } \partial \Omega, \\
{[(1 / \mu) \operatorname{curl} \boldsymbol{A} \wedge \boldsymbol{n}] } & =\mathbf{0}, \\
\operatorname{curl} \boldsymbol{A} & \rightarrow \mu \boldsymbol{H}_{\text {ext }} \quad \text { as } r \rightarrow \infty,
\end{aligned}
$$

where [] denotes the jump in the enclosed quantity across $\partial \Omega$ and $r$ is the distance from the origin. Note that (4) simply states that the current in the exterior region is equal to the applied current (for example, in the solenoid which is generating the applied magnetic field), (5) implies that no supercurrent passes through the boundary, while (6) is the usual boundary condition on the magnetic field at the interface between two media. In fact, since it is physically impossible for the applied current to lie within the sample, we must have curl $\boldsymbol{H}_{e x t}=\mathbf{0}$ in $\Omega$.

We see that there are two important lengthscales associated with (2)-(3). From (2) we see that the natural lengthscale for variations in $\Psi$ is

$$
\xi_{D}=\frac{\hbar}{2 \sqrt{m|a|}},
$$


which is known as the coherence length, while from (3) we see that the natural lengthscale for variations in $\boldsymbol{A}$ (and therefore $\boldsymbol{H}$ ) is

$$
\lambda_{D}=\frac{1}{e} \sqrt{\frac{m b}{2|a| \mu}},
$$

which is known as the penetration depth. The ratio of these two lengthscales is the nondimensional Ginzburg-Landau parameter $\kappa=\lambda_{D} / \xi_{D}$ mentioned in the introduction, which is a key material parameter in determining the behaviour of the superconductor.

We will be concerned only with isothermal conditions below the critical temperature, and for simplicity we will assume that the permeability $\mu$ (and the permittivity $\varepsilon$ ) take the same constant values both inside and outside the superconductor (the modification if this is not the case is easy to make).

We adopt the usual nondimensionalization of the Ginzburg-Landau equations by setting

$$
\Psi=\sqrt{\frac{|a|}{b}} \Psi^{\prime}, \quad \boldsymbol{B}=\mu \boldsymbol{H}=|a| \sqrt{\frac{2 \mu}{b}} \boldsymbol{H}^{\prime}, \quad \boldsymbol{A}=\lambda_{D}|a| \sqrt{\frac{2 \mu}{b}} \boldsymbol{A}^{\prime}, \quad \boldsymbol{x}=\lambda_{D} \boldsymbol{x}^{\prime} .
$$

Then, dropping the primes, we have

$$
\begin{aligned}
\left(\frac{1}{\kappa} \nabla-i \boldsymbol{A}\right)^{2} \Psi & =\left(|\Psi|^{2}-1\right) \Psi \\
-(\operatorname{curl})^{2} \boldsymbol{A} & =\frac{i}{2 \kappa}\left(\Psi^{*} \nabla \Psi-\Psi \nabla \Psi^{*}\right)+|\Psi|^{2} \boldsymbol{A} .
\end{aligned}
$$

Note that the thermodynamic critical field $H_{c}=1 / \sqrt{2}$ in these units.

Ginzburg and Landau's motivation for developing their theory was to determine the surface energy of a superconducting/normal domain boundary, and in particular to show that it was positive. In fact it turns out that this energy is only positive if $\kappa<1 / \sqrt{2}$, i.e. for type-I superconductors. For type-II superconductors the negative surface energy leads to vortex solutions (which we will examine shortly), and the splitting of the thermodynamic critical field $H_{c}$ into a lower critical field $H_{c_{1}}$ and an upper critical field $H_{c_{2}}$, as mentioned in the introduction. In these units the $H_{c_{2}}=\kappa$, while $H_{c_{1}} \sim \frac{\log \kappa}{2 \kappa}$ for large $\kappa$.

Time-dependent versions of the Ginzburg-Landau equations were written down by Schmidt [63], and in 1968 Gor'kov and Eliashberg [38] demonstrated that the following equations (11)-(12) could be derived from the BCS model for a superconductor with paramagnetic impurities. These correspond to a gauge-invariant gradient flow of the Ginzburg-Landau energy functional, and as such are the simplest time-dependent equations whose solutions evolve to the minimisers of that functional. The dimensionless time-dependent Ginzburg-Landau equations are

$$
\begin{aligned}
\frac{1}{\kappa^{2}} \frac{\partial \Psi}{\partial t}+\frac{i \Psi \phi}{\kappa} & =\left(\frac{1}{\kappa} \nabla-i \boldsymbol{A}\right)^{2} \Psi+\Psi\left(1-|\Psi|^{2}\right) \text { in } \Omega, \\
-(\operatorname{curl})^{2} \boldsymbol{A}-\frac{\sigma}{\kappa^{2}}\left(\frac{\partial \boldsymbol{A}}{\partial t}+\nabla \phi\right) & =\frac{i}{2 \kappa}\left(\Psi^{*} \nabla \Psi-\Psi \nabla \Psi^{*}\right)+|\Psi|^{2} \boldsymbol{A} \text { in } \Omega,
\end{aligned}
$$

where $\phi$ is the electric scalar potential, which is such that the electric field $\boldsymbol{E}$ is given by

$$
\boldsymbol{E}=-\frac{\partial \boldsymbol{A}}{\partial t}-\nabla \phi
$$


remember that $\boldsymbol{A}$ is determined only up to a gradient; however, once $\boldsymbol{A}$ is given $\phi$ is determined up to a function of $t$. The constant $\sigma$ is a measure of the normal conductivity of the superconducting material (in this nondimensional form $\sigma$ is the ratio of the timescale for diffusion of magnetic field in the normal state to the timescale for the relaxation of the order parameter).

Outside $\Omega$ we have Maxwell's equations (suitably nondimensionalized, neglecting displacement current)

$$
\begin{aligned}
\operatorname{curl} \boldsymbol{H} & =\boldsymbol{J}_{e x t}, \\
\operatorname{div} \boldsymbol{H} & =0, \\
\boldsymbol{H}_{t}+\operatorname{curl} \boldsymbol{E} & =\mathbf{0}, \\
\operatorname{div} \boldsymbol{E} & =0,
\end{aligned}
$$

where $\boldsymbol{J}_{\text {ext }}$ is the externally imposed current which is driving the system. The most commonly considered situation is that in which a uniform magnetic field is applied at infinity, in which case $\boldsymbol{J}_{\text {ext }}$ is zero and

$$
\boldsymbol{H} \rightarrow \boldsymbol{H}_{\text {ext }} \text { as }|\boldsymbol{x}| \rightarrow \infty
$$

(assuming a bounded superconducting region).

In the time-dependent case we still have the natural boundary condition

$$
\boldsymbol{n} \cdot\left(\frac{1}{\kappa} \nabla-i \boldsymbol{A}\right) \Psi=0 \quad \text { on } \partial \Omega,
$$

along with the usual conditions on Maxwell's equations at an interface, which in the present case of constant permittivity and permeability are the continuity of $\boldsymbol{H}$ and $\boldsymbol{E}$. In addition we may take $\boldsymbol{A}$ and $\phi$ to be continuous across $\partial \Omega$. Note though that these conditions are not all independent, since for example the continuity of the normal component of $\boldsymbol{H}$ arises by taking (15) to hold in a generalised sense everywhere, but this equation is automatic from (1), which, if it holds in a generalised sense, gives continuity of the tangential component of $\boldsymbol{A}$.

A commonly considered special case is the two-dimensional situation in which $\Omega$ is the cylinder $D \times \mathbb{R}, D \subseteq \mathbb{R}^{2}$, with an applied magnetic field $\boldsymbol{H} \equiv\left(0,0, H_{\text {ext }}\right)$ as $x^{2}+y^{2} \rightarrow \infty$. In this case the solutions of (11)-(12) are of the form

$$
\begin{aligned}
\boldsymbol{H} & =(0,0, H(x, y, t)), \\
\boldsymbol{A} & =\left(A_{1}(x, y, t), A_{2}(x, y, t), 0\right),
\end{aligned}
$$

and the Maxwell equations

$$
\operatorname{curl} \boldsymbol{H}=\mathbf{0}, \quad \operatorname{div} \boldsymbol{H}=0,
$$

outside $\Omega$ imply $\nabla H=\mathbf{0}$, so that $H \equiv H_{\text {ext }}$ outside $\Omega^{2}$. Thus the continuity of magnetic field across $\partial \Omega$ leads to the Dirichlet condition $H=H_{\text {ext }}$ on $\partial D$, and we do not have to consider the region external to the superconductor further. We note also that the potential $\boldsymbol{A}$ can be chosen so that

$$
\boldsymbol{A} \cdot \boldsymbol{n}=0 \quad \text { on } \partial D .
$$

We will return to this two-dimensional situation often throughout the paper, since it is usually much simpler than the general case.

\footnotetext{
${ }^{2}$ If $\Omega$ is not simply connected then $H \equiv H_{\text {ext }}$ in the unbounded component of $\mathbb{R}^{3} \backslash \Omega$, but in each bounded component of $\mathbb{R}^{3} \backslash \Omega$ (i.e. in the holes of $\Omega$ ) all we can say is $H=$ constant. These constants must be determined as part of the solution.
} 
Equations (11)-(12) are gauge invariant in the sense that they are invariant under transformations of the form

$$
\boldsymbol{A} \rightarrow \boldsymbol{A}+\nabla \omega, \quad \phi \rightarrow \phi-\frac{\partial \omega}{\partial t}, \quad \Psi \rightarrow \Psi e^{i \kappa \omega} .
$$

We may take advantage of this invariance to write the equations in terms of real variables by writing

$$
\Psi=f e^{i \chi}, \quad \boldsymbol{Q}=\boldsymbol{A}-\frac{1}{\kappa} \nabla \chi, \quad \Phi=\phi+\frac{1}{\kappa} \frac{\partial \chi}{\partial t},
$$

to give

$$
\begin{aligned}
-\frac{1}{\kappa^{2}} \frac{\partial f}{\partial t}+\frac{1}{\kappa^{2}} \nabla^{2} f & =f^{3}-f+f|\boldsymbol{Q}|^{2} \quad \text { in } \Omega, \\
f^{2} \Phi+\operatorname{div}\left(f^{2} \boldsymbol{Q}\right) & =0 \quad \text { in } \Omega, \\
-(\operatorname{curl})^{2} \boldsymbol{Q} & =\frac{\sigma}{\kappa^{2}}\left(\frac{\partial \boldsymbol{Q}}{\partial t}+\nabla \Phi\right)+f^{2} \boldsymbol{Q} \quad \text { in } \Omega \\
\boldsymbol{H} & =\operatorname{curl} \boldsymbol{Q}, \\
\boldsymbol{E} & =-\frac{\partial \boldsymbol{Q}}{\partial t}-\nabla \Phi .
\end{aligned}
$$

Equations (11)-(12) exhibit vortex solutions, which can be illustrated by seeking a solution of the form

$$
\begin{gathered}
\Psi=f(r) e^{i n \theta}, \\
\boldsymbol{A}=A(r) \boldsymbol{e}_{\theta},
\end{gathered}
$$

on an infinite domain, where $n$ is an integer known as the vortex number, $r$ and $\theta$ are polar coordinates, and $\boldsymbol{e}_{\theta}$ is the unit vector in the azimuthal direction. Substituting into (11)-(12) gives

$$
\begin{aligned}
& \frac{1}{\kappa^{2}} \frac{1}{r} \frac{d}{d r}\left(r \frac{d f}{d r}\right)-\left(A-\frac{n}{\kappa r}\right)^{2} f=f^{3}-f, \\
& \frac{d}{d r}\left(\frac{1}{r} \frac{d}{d r}(r A)\right)=f^{2}\left(A-\frac{n}{\kappa r}\right), \\
& f, \quad A \text { bounded } \quad \text { as } \quad r \rightarrow 0, \\
& f \rightarrow 1, \quad A \rightarrow 0 \quad \text { as } \quad r \rightarrow \infty .
\end{aligned}
$$

The existence of a unique solution which necessarily has $f(0)=0$ has been shown by Berger and Chen [9]. The supercurrent is given by

$$
\boldsymbol{J}=\operatorname{curl} \boldsymbol{H}=-f^{2}\left(A-\frac{n}{\kappa r}\right) \boldsymbol{e}_{\theta},
$$

which shows the vortex nature of this solution. The axial magnetic field carried by the vortex is

$$
\int_{\mathbb{R}^{2}} \boldsymbol{H} \cdot \boldsymbol{d} \boldsymbol{S}=\frac{2 \pi n}{\kappa}
$$

which is quantized in units of $2 \pi / \kappa$, with $n$ the number of flux quanta carried by the vortex. Note that for large values of $\kappa, f \approx 1$ except in a region of order $\kappa^{-1}$ from the origin, which is the vortex core. 
Since the flux quantum is $O(1 / \kappa)$ it is common to rescale magnetic field with $1 / \kappa$ when considering vortex solutions. We set

$$
\boldsymbol{H}=\frac{\overline{\boldsymbol{H}}}{\kappa}, \quad \boldsymbol{A}=\frac{\overline{\boldsymbol{A}}}{\kappa}, \quad \phi=\frac{\bar{\phi}}{\kappa}, \quad \boldsymbol{E}=\frac{\overline{\boldsymbol{E}}}{\kappa}, \quad \boldsymbol{J}=\frac{\overline{\boldsymbol{J}}}{\kappa}, \quad \boldsymbol{Q}=\frac{\overline{\boldsymbol{Q}}}{\kappa}, \quad \Phi=\frac{\bar{\Phi}}{\kappa},
$$

to give, on dropping the bars,

$$
\begin{aligned}
-\frac{\partial f}{\partial t}+\nabla^{2} f & =\kappa^{2}\left(f^{3}-f\right)+f|\boldsymbol{Q}|^{2} \quad \text { in } \Omega, \\
f^{2} \Phi+\operatorname{div}\left(f^{2} \boldsymbol{Q}\right) & =0 \quad \text { in } \Omega, \\
-(\operatorname{curl})^{2} \boldsymbol{Q} & =\frac{\sigma}{\kappa^{2}}\left(\frac{\partial \boldsymbol{Q}}{\partial t}+\nabla \Phi\right)+f^{2} \boldsymbol{Q} \quad \text { in } \Omega, \\
\boldsymbol{H} & =\operatorname{curl} \boldsymbol{Q}, \\
\boldsymbol{E} & =-\frac{\partial \boldsymbol{Q}}{\partial t}-\nabla \Phi .
\end{aligned}
$$

The literature on the Ginzburg-Landau model is vast and various, both in the mathematics and physics communities, and this is not the place to delve into it. We note though the mathematical reviews of the model in $[17,31,41]$, the pioneering work on the vortex state by Abrikosov [1], and the numerical simulations of $[23,29,30,40,42]$.

\subsection{Vortex Pinning in the Ginzburg-Landau model}

In most technological applications superconductors are required to carry a transport current. The interaction of this current with the current circling a vortex causes the vortex to move (this is often considered to be the result of the "Lorentz force" on the magnetic flux line carried by the vortex due to the transport current). The motion of the vortex dissipates energy, leads to an electric field, and hence a nonzero resistivity, and is therefore undesirable. In practice attempts are made to "pin" vortices at certain sites in the material in order to impede their motion. It is found that any form of inhomogeneity (for example impurities, dislocations or grain boundaries) will help to pin vortices. Such impurities have the effect of impeding locally the ability of the material to become superconducting. A popular way of modelling this inhomogeneity in the Ginzburg-Landau framework is to allow the equilibrium density of superconducting electrons to vary spatially $[45,15]$. In the simplest case this leads to

$$
\begin{aligned}
\frac{\partial \Psi}{\partial t}+i \Psi \phi & =(\nabla-i \boldsymbol{A})^{2} \Psi+\kappa^{2} \Psi\left(a(\boldsymbol{x})-|\Psi|^{2}\right) \text { in } \Omega, \\
-(\operatorname{curl})^{2} \boldsymbol{A}-\frac{\sigma}{\kappa^{2}}\left(\frac{\partial \boldsymbol{A}}{\partial t}+\nabla \phi\right) & =\frac{i}{2}\left(\Psi^{*} \nabla \Psi-\Psi \nabla \Psi^{*}\right)+|\Psi|^{2} \boldsymbol{A} \text { in } \Omega,
\end{aligned}
$$

where the equilibrium density of superconducting electrons is denoted by $a(\boldsymbol{x})$. Of course, more generally we may allow the coefficient of $|\Psi|^{2} \Psi$ as well as $\lambda_{D}, \xi_{D}, \kappa$ and $\sigma$ to vary spatially, but we will consider here the case when these parameters are constant. As before, we may use the relationships (20) to write the equations in terms of real variables as

$$
\begin{aligned}
-\frac{\partial f}{\partial t}+\nabla^{2} f & =\kappa^{2} f\left(f^{2}-a(\boldsymbol{x})\right)+f|\boldsymbol{Q}|^{2} \text { in } \Omega, \\
f^{2} \Phi+\operatorname{div}\left(f^{2} \boldsymbol{Q}\right) & =0 \text { in } \Omega,
\end{aligned}
$$




$$
\begin{aligned}
-(\operatorname{curl})^{2} \boldsymbol{Q} & =\frac{\sigma}{\kappa^{2}}\left(\frac{\partial \boldsymbol{Q}}{\partial t}+\nabla \Phi\right)+f^{2} \boldsymbol{Q} \text { in } \Omega, \\
\boldsymbol{H} & =\operatorname{curl} \boldsymbol{Q} \\
\boldsymbol{E} & =-\frac{\partial \boldsymbol{Q}}{\partial t}-\nabla \Phi .
\end{aligned}
$$

Numerical simulations of these equations in two dimensions show that the vortices are attracted to minima of $a$, as we would have hoped $[15,26]$.

\section{The London equation}

Numerical solution of the Ginzburg-Landau model is costly, and becomes more difficult as the number of vortices increases and also as the core radius of the vortices, which we have seen is $1 / \kappa$ in these units, becomes small. The value of $\kappa$ is typically quite small for pure metals, but may be quite large for superconducting alloys. Examples include $\mathrm{NbSn}$ with $\kappa \approx 15.3$, NbNi with $\kappa \approx 28.0$ and $\mathrm{V}_{3} \mathrm{G}$ with $\kappa \approx 25.3$. In addition, all the high-temperature superconducting materials have very large values of $\kappa$, in the range 50-100 (although it is still not clear if the GL model is applicable to high-temperature materials).

While large values of $\kappa$ are a hindrance to numerical simulations, they allow us to simplify the equations by considering the asymptotic limit of the Ginzburg-Landau model in which $\kappa \rightarrow \infty$, which leads to what is known as the London model. Since the vortex cores have a radius of order $1 / \kappa$, they become vanishingly small in this limit, and the vortices become line singularities. We see that equation (34) implies that $f=1$ except at these isolated vortex lines. Then equation (36) becomes

$$
(\operatorname{curl})^{2} \boldsymbol{Q}+\boldsymbol{Q}=\mathbf{0},
$$

except on the vortex lines. Taking the curl of this equation we find

$$
(\operatorname{curl})^{2} \boldsymbol{H}+\boldsymbol{H}=\mathbf{0},
$$

which is the London equation in the absence of vortices, and is valid away from vortex lines.

This equation was first written down in 1935 by F. and H. London [47, 46] (and thus predates the Ginzburg-Landau model). The aim was not to describe vortices (whose existence was not established at the time) but to describe the Meissner effect of the exclusion of magnetic field from a bulk superconducting sample except in a thin surface penetration layer. Equation (47) predicts an exponential decay of the magnetic field away from the boundary of the sample, over a lengthscale of the penetration depth. Note that with $f=1$ equations (42) and (46) imply that $\Phi=0$, so that $(45)$ is

$$
\boldsymbol{E}=\frac{\partial}{\partial t}(\operatorname{curl} \boldsymbol{H})=\frac{\partial \boldsymbol{J}}{\partial t} .
$$

This equation was also written down by London \& London $[47,46]$. It states that an electric field will accelerate superconducting electrons rather than simply maintaining their speed in the presence of dissipation as in Ohm's law, and so describes the perfect superconductivity of the supercurrent.

Returning to our asymptotic expansion of the Ginzburg-Landau model, we note that the magnetic field will be singular as each vortex line is approached; we need to look locally at the core of each vortex to determine the nature of the singularity, which will then become a matching condition on equation (47). 
Let a vortex lie along the curve $\Gamma$, given by $\boldsymbol{x}=(x, y, z)=\boldsymbol{q}(s, t)$, where $s$ measures arclength along the curve. We define a local curvilinear coordinate system $(s, \rho, \theta, T)$ by

$$
\begin{aligned}
\boldsymbol{x} & =\boldsymbol{q}(s, t)+\rho \cos \theta \boldsymbol{n}(s, t)+\rho \sin \theta \boldsymbol{b}(s, t), \\
t & =T
\end{aligned}
$$

where $\boldsymbol{n}$ is the unit normal and $\boldsymbol{b}$ is the unit binormal to the curve $\Gamma$. We may look locally near the vortex core by introducing the stretched variable $R=\kappa \rho$. Then, providing the radius of curvature of the vortex is much greater than the core radius $1 / \kappa$, the vortex will appear rectilinear to leading order in the inner region.

The solution of the Ginzburg-Landau equations in this inner region to leading order is [18]

$$
\begin{aligned}
\chi & =\theta, \\
\boldsymbol{Q} & =-\frac{\kappa}{R} \boldsymbol{e}_{\theta}, \\
f & =f(R),
\end{aligned}
$$

where $f(R)$ satisfies

$$
\begin{aligned}
f^{\prime \prime}+\frac{1}{R} f^{\prime}-\frac{1}{R^{2}} f & =f^{3}-f, \\
f(0) & =0, \\
f(\infty) & =1,
\end{aligned}
$$

and we have assumed that the vortex has unit vortex number, since this is thought to be the only stable case. It is proved in [22] that the solution to (54)-(56) exists and is unique. Matching (52) with the outer solution means that the outer $\boldsymbol{Q}$ must have the asymptotic behaviour

$$
\boldsymbol{Q} \sim-\frac{1}{\rho} \boldsymbol{e}_{\theta} \text { as } \rho \rightarrow 0 .
$$

Equations (37) and (46) imply that $\operatorname{curl} \boldsymbol{H}=-\boldsymbol{Q}$ in the outer region. This, together with (57), implies that $\boldsymbol{H}$ must have the asymptotic behaviour

$$
\boldsymbol{H} \sim-\log \rho \boldsymbol{t} \text { as } \rho \rightarrow 0
$$

as the vortex line is approached, where $\boldsymbol{t}$ is the unit tangent to the vortex line. This behaviour may be conveniently expressed by writing (47) as

$$
(\operatorname{curl})^{2} \boldsymbol{H}+\boldsymbol{H}=2 \pi \boldsymbol{\delta}_{\Gamma}(\boldsymbol{x}),
$$

where

$$
\boldsymbol{\delta}_{\Gamma}(\boldsymbol{x})=\int_{\Gamma} \delta(x-\bar{x}) \delta(y-\bar{y}) \delta(z-\bar{z}) \boldsymbol{d} \overline{\boldsymbol{x}} .
$$

Equation (59) now holds in a generalised sense in the whole superconducting region. There is a nice analogy here between superconducting vortices in the London equation and line vortices in an inviscid, incompressible and otherwise irrotational fluid. The fluid velocity $\boldsymbol{u}$ is analogous to the electric current $\boldsymbol{J}$, while the magnetic field $\boldsymbol{H}$ is analogous to the vector streamfunction. This analogy is explored in more detail in [13]. 
By proceeding to the next order in the inner expansion, a law of motion for superconducting vortices can be derived, which up to and including order-one terms is [18]

$$
\frac{\beta}{2} \boldsymbol{v}=\frac{C \log \kappa}{2} \boldsymbol{n}+\boldsymbol{J} \wedge \boldsymbol{t},
$$

where $C$ is the curvature of the vortex line, $\beta / 2$ is an order-one mobility which depends on the parameter $\sigma$ in the Ginzburg-Landau equations, and $\boldsymbol{J}$ is the regular part of the outer current evaluated at the vortex line, which includes the contribution from the "arms" of the vortex $\Gamma$ as well as contributions from other vortices, boundaries, and applied currents. This equation was first derived for almost rectilinear vortices by Gor'kov \& Kopkin [39]. Their analysis was put into a more mathematical framework by Peres \& Rubinstein in [50], using techniques developed by Neu [49]. The law for curved vortices was derived independently by E [32] and Chapman \& Richardson [18] and an analogous derivation using a complex relaxation time for the order parameter to describe the Hall effect was given by Dorsey in [27].

Clearly we may rescale time so that the mobility of the vortex is unity, and it is common to do so. Thus by means of our asymptotic analysis we have reduced the coupled nonlinear time-dependent equations of the Ginzburg-Landau model to the linear, quasi-static, but singular equation (59) and the law of motion (61). The great advantage of the linearity of (59) is that the contributions to the magnetic field of many different vortices can now simply be added together through the principle of superposition; the equation for $n$ vortices is simply

$$
(\operatorname{curl})^{2} \boldsymbol{H}+\boldsymbol{H}=2 \pi \sum_{k=1}^{n} \boldsymbol{\delta}_{\Gamma_{k}}(\boldsymbol{x}) .
$$

The interaction between vortices all takes place through the law of motion (61).

To close the system (61)-(62) we need to add Maxwell's equations

$$
\operatorname{curl} \boldsymbol{H}=\mathbf{0}, \quad \operatorname{div} \boldsymbol{H}=0,
$$

outside $\Omega$ with $\boldsymbol{H}$ continuous across $\partial \Omega$ and

$$
\boldsymbol{H} \rightarrow \boldsymbol{H}_{\text {ext }}
$$

at infinity. It has been shown in [16] that (61), (62) imply that vortices must meet the boundary $\partial \Omega$ normally, since, if this is not so, an infinite current density is produced (this can also be thought of as an infinite curvature of the vortex and its image, which is clearly incompatible with (61)).

\subsection{Two dimensions}

In the usual two-dimensional setting with $\boldsymbol{H}=(0,0, H(x, y, t))$ the vortices are all rectilinear and normal to the plane of interest, so that there are no curvature terms in the law of motion, and (61) and (62) become

$$
\begin{aligned}
-\nabla^{2} H+H & =2 \pi \sum_{k=1}^{n} z_{k} \delta\left(\boldsymbol{x}-\boldsymbol{x}_{k}\right) \text { in } D, \\
\dot{\boldsymbol{x}}_{k} & =-z_{k} \nabla H,
\end{aligned}
$$

where $\boldsymbol{x}_{k}$ is the position in the plane of the $k$ th vortex, $z_{k}$ is either \pm 1 depending on whether the vortex is up or down, a dot represents $d / d t$, and the right-hand side of (65) represents the regular part of the gradient of $H$. To these we must add the boundary condition

$$
H=H_{\text {ext }} \quad \text { on } \partial D .
$$


Note in particular that these equations imply that like vortices repel each other, opposite vortices attract each other. This is in contrast to fluid vortices in two dimensions, in which the interaction force is perpendicular to the line of centres.

\subsection{Vortex Pinning in the London Model}

A similar asymptotic analysis can be carried out in the presence of a varying equilibrium density of superconducting electrons as in (39)-(40). In this case the London equation becomes

$$
\operatorname{curl}\left(\frac{1}{a(\boldsymbol{x})} \operatorname{curl} \boldsymbol{H}\right)+\boldsymbol{H}=2 \pi \boldsymbol{\delta}_{\Gamma}(\boldsymbol{x}),
$$

where $\boldsymbol{\delta}_{\Gamma}(\boldsymbol{x})$ is as before, while the law of motion is modified to [19]

$$
\frac{\beta}{2} \boldsymbol{v}=\frac{C \log \kappa}{2} \boldsymbol{n}+\frac{1}{a} \boldsymbol{J} \wedge \boldsymbol{t}-\frac{\log \kappa}{2} \nabla \log a .
$$

Thus there is an extra "pinning potential" term which must be added to the usual current driving forces. We see that the vortices are attracted to the minima of $a$.

The effectiveness of the pinning potential depends on the relative magnitude of the terms in (68). In particular we note that if the equilibrium density of superconducting electrons, $a$, varies on a lengthscale $\delta$ (by comparison to the penetration depth), then the magnitude of the pinning force is $(1 / \delta) \log \kappa$.

\section{Vortex-density models}

Typically there are many millions of vortices present in a laboratory superconducting sample in a magnetic field. While the London model is simpler than the Ginzburg-Landau model, it is still prohibitively expensive to keep track of millions of individual vortices, and it becomes appealing to seek a model describing the evolution of a vortex density, rather like the vorticity in fluid mechanics.

Starting from the London model

$$
\begin{aligned}
(\operatorname{curl})^{2} \boldsymbol{H}+\boldsymbol{H} & =2 \pi \sum_{i} \boldsymbol{\delta}_{\Gamma_{i}}(\boldsymbol{x}), \\
\operatorname{div} \boldsymbol{H} & =0,
\end{aligned}
$$

let us consider a limit in which the vortex separation, $\nu$ say, is much less than unity, while still being much greater than the core radius of the vortices (remember we are on the lengthscale of the penetration depth). Following [13] we formally define the vortex density $\boldsymbol{\omega}$ as

$$
\boldsymbol{\omega}=\lim _{\eta \rightarrow 0} \nu^{2} \int_{|\boldsymbol{x}-\overline{\boldsymbol{x}}|<\eta} \frac{3}{2 \eta^{3}} \sum_{i} \boldsymbol{\delta}_{\Gamma_{i}}(\overline{\boldsymbol{x}}) d \overline{\boldsymbol{x}}
$$

and rescale the magnetic field and electric current with $1 / \nu^{2}$. Then formally, as $\kappa \rightarrow \infty$ and $\eta \rightarrow 0$, with $1 / \kappa \ll \nu \ll \eta \ll 1$, by integrating (69)-(70) over the region $|\boldsymbol{x}-\overline{\boldsymbol{x}}|<\eta$, we have

$$
\begin{aligned}
(\operatorname{curl})^{2} \boldsymbol{H}+\boldsymbol{H} & =\boldsymbol{\omega}, \\
\operatorname{div} \boldsymbol{H} & =0,
\end{aligned}
$$

where, with a slight abuse of notation, we have now used $\boldsymbol{H}$ to denote the local average of the magnetic field. To these equations we must add an equation to describe conservation of vortices, 
which is automatic when keeping track of isolated individual vortices, but has been lost in the averaging process. Such an equation is derived by noting that the number of vortices crossing an arbitrary open surface $S$ convected with the same velocity field as the vortices must be constant [13]. This gives

$$
\frac{d}{d t} \int_{S(t)} \sum_{i} \boldsymbol{\delta}_{\Gamma_{i}}(\boldsymbol{x}) \cdot \boldsymbol{d} \boldsymbol{S}=0
$$

which may be averaged to give the integral conservation law

$$
\frac{d}{d t} \int_{S(t)} \boldsymbol{\omega} \cdot \boldsymbol{d} \boldsymbol{S}=\mathbf{0}
$$

Since $S$ is arbitrary there is an equivalent differential conservation law,

$$
\frac{\partial \boldsymbol{\omega}}{\partial t}+\operatorname{curl}(\boldsymbol{\omega} \wedge \boldsymbol{v})=0,
$$

where $\boldsymbol{v}$ is the velocity field of the vortices.

We note that the electric field is given by [18]

$$
\boldsymbol{E}=\boldsymbol{J}_{t}+\boldsymbol{\omega} \wedge \boldsymbol{v}
$$

The first term on the right-hand side is the London term (48). The second term describes the electric field generated by the moving magnetic field attached to vortices as they translate. We see from (72) and (77) that (76) is actually the Faraday law (16).

To close the model we need to give a relationship for $\boldsymbol{v}$. This can be derived from an inner analysis of a vortex core in the same manner as for isolated vortices. The result is [18]

$$
\boldsymbol{v}=\boldsymbol{J} \wedge \hat{\boldsymbol{\omega}}+\epsilon \operatorname{curl} \hat{\boldsymbol{\omega}} \wedge \hat{\boldsymbol{\omega}}
$$

where $\epsilon=\left(\nu^{2} \log \kappa\right) / 2, \hat{\boldsymbol{\omega}}$ is the unit vector in the direction of $\boldsymbol{\omega}$ (throughout we will use a hat over a vector to signify the unit vector in the same direction), the curvature $C$ has been written as $\operatorname{curl} \hat{\boldsymbol{\omega}} \wedge \hat{\boldsymbol{\omega}}$, and time has been scaled by $\nu^{2} \beta / 2$, so that the coefficient of the first term on the right-hand side of $(78)$ is unity.

We see that if $\nu^{2} \log \kappa \ll 1$ the motion of the vortices is dominated by the average current density; the self-induced motion becomes a lower-order correction. It is tempting in this limit to neglect the self-induced contribution altogether, which indeed was done when the model was first derived in [13]. However, a linear stability analysis of a single rectilinear vortex under the law of motion (78) gives the dispersion relation [55, 24]

$$
\varsigma=|\boldsymbol{J} \cdot \hat{\boldsymbol{\omega}}| k-\epsilon k^{2},
$$

where $\varsigma$ is the growth rate and $k$ is the wavenumber of the perturbation. Thus if $\epsilon$ is set to zero the growth rate is unbounded whenever the component of $\boldsymbol{J}$ in the direction of the vortex line is non-zero. The reason for this instability is that a small high-frequency oscillation on the vortex can lead to an order-one change in the angle of the vortex line, which produces an order-one change in the velocity of the vortex. The self-induced term (i.e. the second term in (78)) regularises the singularity by damping oscillations of very high-frequency. The most unstable mode then has wavenumber $|\boldsymbol{J} \cdot \hat{\boldsymbol{\omega}}| /(2 \epsilon)$ and a growth rate of $|\boldsymbol{J} \cdot \hat{\boldsymbol{\omega}}|^{2} /(4 \epsilon)$. Two examples of the evolution of this mode for different background currents are shown in Figure 4. We see that the vortex line becomes 

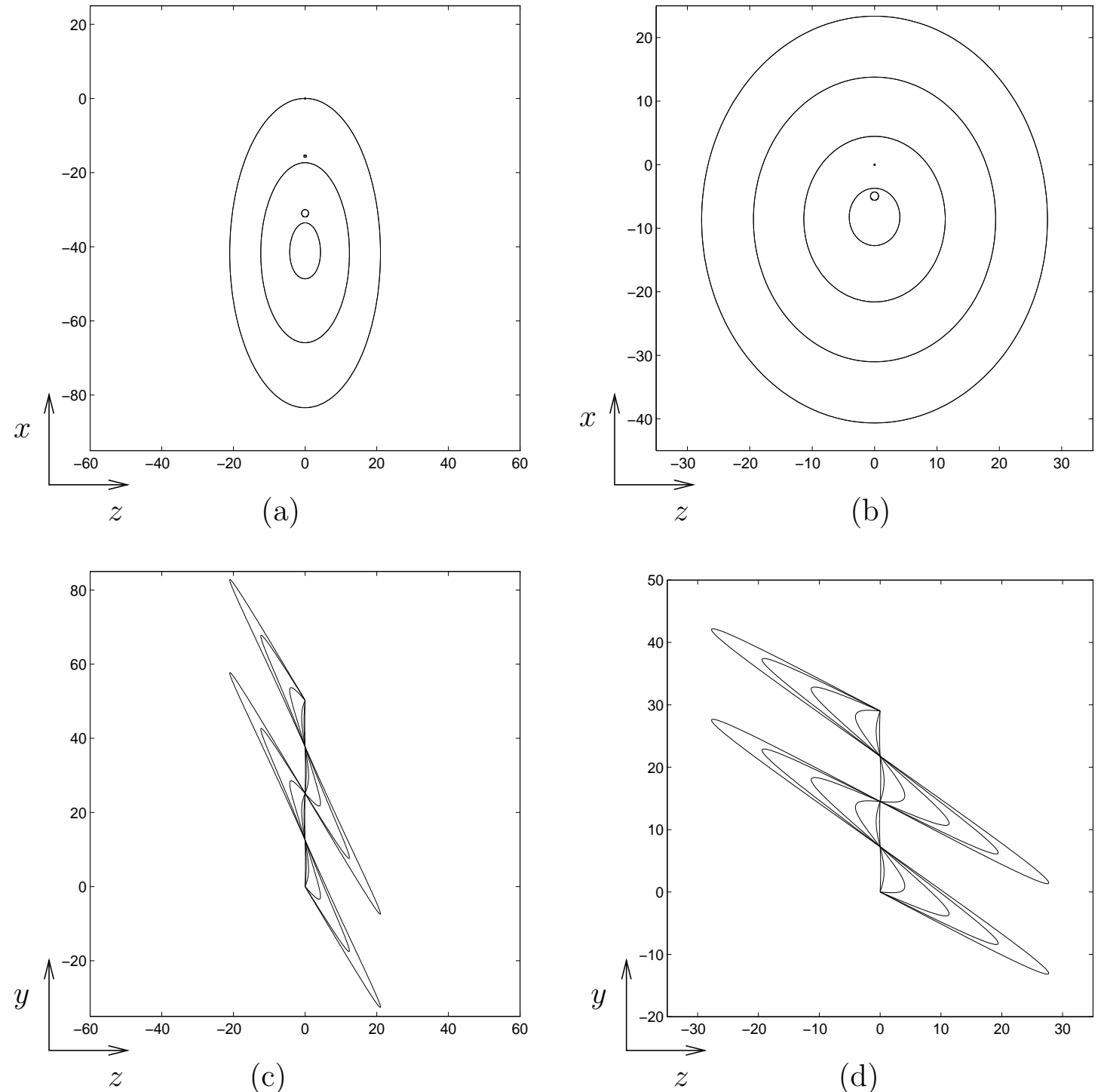

Figure 4: The evolution of the most unstable mode of the helical instability for a single vortex under the influence of a constant background current. The unperturbed vortex lies parallel to the $y$-axis and translates in the $x$-direction. Plots (a) and (c) show the case $\boldsymbol{J}=(0,1 / 2, \sqrt{3} / 2)$ at times $t=$ $0,18,36, \cdots, 90$. Plots (b) and (d) show the case $\boldsymbol{J}=(0, \sqrt{3} / 2,1 / 2)$ at times $t=0,10,20, \cdots, 50$. Figure due to G. Richardson. 
a helix, which evolves in such a way as to try to make the vortex perpendicular to the background current.

The question arises as to the repercussions of this single-vortex instability on the vortex-density model (72), (73), (76), (78). The analysis in [56] indicates that if the self-induced term is neglected the model may be ill-posed, and hence although the self-induced term is small it may be necessary to retain it as a regularising mechanism. However, as we can see from the single-vortex case, the lengthscale of the regularised instability is small (indeed the wavelength of the instability may be less than the vortex separation), and therefore it may be more appealing to try to model the largescale effects of this small-scale instability, rather than including a small-scale regularisation in the vortex-density model. We will attempt to do this in Section 5.

For the moment we will concentrate on situations in which $\boldsymbol{J} \cdot \boldsymbol{\omega} \equiv 0$, which include the twodimensional situations of a cylinder in an axial or transverse magnetic field, and three-dimensional situations with axial symmetry. In this case we may take the limit $\epsilon \rightarrow 0$ and replace (78) by

$$
\boldsymbol{v}=\boldsymbol{J} \wedge \hat{\boldsymbol{\omega}}
$$

The mathematical analysis of (72), (73), (76) and (78) is in its infancy, but some results are beginning to appear in the two-dimensional settings of a cylinder in an axial or transverse magnetic field, which we discuss in $\S 4.3$ below.

Note that equation (69) (derived originally for $|\boldsymbol{H}|=O(1)$ ) is valid so long as $|\boldsymbol{H}|=o(\kappa)$, since this gives $|\boldsymbol{Q}|=o(\kappa)$ and therefore $f=1+o(1)$. Thus our vortex-density model (72) is limited to magnetic fields $1 / \kappa \ll\left|\boldsymbol{H}_{\text {ext }}\right| \ll 1$ in the original scaling ${ }^{3}$. This corresponds to limiting the separation between vortices $\nu$ to be such that $\kappa^{-1 / 2} \ll \nu \ll 1$. However, a vortex-density model can be derived for larger magnetic fields, from a direct multiple scales analysis of the Ginzburg-Landau equations, rather than from the London model [57]. At higher magnetic fields, to maintain $f$ close to unity between the vortex cores, the magnetic field is forced to vary over a longer lengthscale than the penetration depth, and the resulting model is equivalent to the long-wave approximation of the present model, which we discuss in Section 4.4. Note in this connection that a stability analysis in [14] shows that any solution in which $f<(2 / 3)^{1 / 2}$ away from vortex cores is unstable. It is likely that if a large gradient in $\boldsymbol{H}$ did exist, forcing $f$ away from unity, then this instability will lead to the formation of more vortices, which will reduce the gradient of $\boldsymbol{H}$ and allow $f$ to return to unity.

\subsection{Relationship with the hydrodynamic model of Marchetti and Nelson}

It is useful to compare the model (72), (73), (76) and (78) with that introduced by Marchetti and Nelson in [48]. They imagine a situation in which the applied magnetic field is in the $z$-direction and introduce the areal vortex density $n_{v}(\boldsymbol{r}, t)$ and the two components of a tangent field $\boldsymbol{\tau}(\boldsymbol{r}, t)$ describing the instantaneous tile of the vortex lines, which are given by

$$
\begin{aligned}
n_{v} & =\sum_{i} \delta\left(\boldsymbol{r}_{\perp}-\boldsymbol{r}_{i}(z, t)\right), \\
\boldsymbol{\tau} & =\sum_{i} \frac{\partial \boldsymbol{r}_{i}}{\partial z} \delta\left(\boldsymbol{r}_{\perp}-\boldsymbol{r}_{i}(z, t)\right),
\end{aligned}
$$

\footnotetext{
${ }^{3}$ Remember that in $(72)$ we have rescaled the magnetic field first with $1 / \kappa$ and then with $1 / \nu^{2}$ so that $\boldsymbol{H}$ stays order one as $\kappa \rightarrow \infty$. However, it is useful during the discussion of the models to have some absolute measure of the size of the magnetic field, and for this we use the original scalings for the Ginzburg-Landau equations (11), (12). In particular this makes it easier to compare the magnitude of the magnetic field in each model to the critical magnetic fields $H_{c}, H_{c_{1}}$ and $H_{c_{2}}$.
} 
where $\boldsymbol{r}=\left(\boldsymbol{r}_{\perp}, z\right), \boldsymbol{r}_{i}$ is a two-dimensional vector denoting the position of the $i$ th vortex in the $x y$ plane, and $\delta$ is the two-dimensional Dirac $\delta$-function. Since vortices are only created or destroyed at boundaries they have the continuity equation

$$
\frac{\partial n_{v}}{\partial t}+\nabla_{\perp} \cdot\left(n_{v} \boldsymbol{v}\right)
$$

where $\boldsymbol{v}$ is the vortex velocity. For the dynamics of the tangent field they set

$$
\frac{\partial \tau_{i}}{\partial t}+\frac{\partial j_{i j}^{T}}{\partial x_{j}}=\frac{\partial\left(n_{v} v_{j}\right)}{\partial z}
$$

where $j_{i j}^{T}$ is the "antisymmetric tangent flux tensor", and the summation convention is used with $x_{1} \equiv x, x_{2} \equiv y$. Finally, since flux lines cannot stop inside the sample, they have a continuity equation in their timelike variable $z$

$$
\frac{\partial n_{v}}{\partial z}+\nabla_{\perp} \cdot \boldsymbol{\tau}=0
$$

To close the model they give constitutive relations for $j_{i j}^{T}$ and $\boldsymbol{v}$. The antisymmetric tangent flux tensor is chosen as

$$
j_{i j}^{T}=\Gamma\left(\frac{\partial \tau_{j}}{\partial x_{i}}-\frac{\partial \tau_{i}}{\partial x_{j}}\right),
$$

where $\Gamma$ is a "kinematic constant", while $\boldsymbol{v}$ is obtained from a force balance involving frictional resistance to vortex motion, a reversible force due to changes in the free energy of the vortex liquid, viscous forces due to flux line entanglement, and an applied transport current as a body force. Finally, the equations in [48] have all been linearised about the state $n_{v}=n_{v}^{0}, \boldsymbol{v}=\boldsymbol{\tau}=\mathbf{0}$.

Let us briefly see how this model relates to the model (72), (73), (76) and (78). First we note that Marchetti and Nelson are linearising about a state in which the vortices are all aligned in the $z$-direction (hence their "time-like" variable $z$ ), whereas we are allowing an arbitrary vortex configuration, and so have no preferred coordinate direction. In fact our vortex density $\boldsymbol{\omega}$ corresponds simply to

$$
\boldsymbol{\omega}=\left(\tau_{1}, \tau_{2}, n_{v}\right) .
$$

Thus their continuity equation (85) in the time-like variable $z$ is

$$
\nabla \cdot \omega=0,
$$

which follows from (72) and (73). In the notation of Marchetti and Nelson the third component the law of vortex conservation $(76)$ is

$$
\frac{\partial n_{v}}{\partial t}+\frac{\partial}{\partial x}\left(n_{v} v_{1}-\tau_{1} v_{3}\right)+\frac{\partial}{\partial y}\left(n_{v} v_{2}-\tau_{2} v_{3}\right)=0
$$

Now any component of $\boldsymbol{v}$ in the direction of the tangent to the vortex has no effect of the motion of the line. We chose $\boldsymbol{v}$ to be orthogonal to the vortex, but since Marchetti and Nelson are considering the position of vortices as points moving in a plane, they have chosen $\boldsymbol{v}$ to lie in the $x y$-plane. So long as the vortex itself does not lie in the $x y$-plane we may, without loss of generality, choose $v_{3}=0$. Equation (89) then corresponds to (83). 
Now let us consider the dynamics of the tangent field. The other two components of (76), in the notation of Marchetti and Nelson, are

$$
\begin{aligned}
\frac{\partial \tau_{1}}{\partial t}+\frac{\partial}{\partial y}\left(\tau_{1} v_{2}-\tau_{2} v_{1}\right) & =\frac{\partial}{\partial z}\left(n_{v} v_{1}-\tau_{1} v_{3}\right) \\
\frac{\partial \tau_{2}}{\partial t}-\frac{\partial}{\partial x}\left(\tau_{1} v_{2}-\tau_{2} v_{1}\right) & =\frac{\partial}{\partial z}\left(n_{v} v_{2}-\tau_{2} v_{3}\right)
\end{aligned}
$$

Hence, with $v_{3}=0$, these correspond to (84), providing

$$
j_{12}^{T}=\tau_{1} v_{2}-\tau_{2} v_{1} .
$$

However, in our case $j_{12}^{T}$ is determined by the principle of vortex conservation, and is not a constitutive relation; (92) is quite different to (86). In fact, in the linearised regime of Marchetti and Nelson (92) gives $j_{12}^{T}=0$ at leading order.

Finally, let us comment on the two different velocity laws. The law (78) arises from a balance of the "friction" during vortex motion with the Lorentz force due to the local current density; the mutual interaction between vortices all takes place through their associated magnetic fields. The mutual interaction in the model of Marchetti and Nelson is modelled through a combination of the free-energy of a flux line liquid and "viscosity" terms due to flux line entanglement. In (72), (73), (76), (78) if a transport current is applied it is applied at the boundary and appears as a boundary condition; the current inside the superconductor is determined from the equations. In contrast the uniform transport current of Marchetti and Nelson is applied throughout the sample.

\subsection{Boundary conditions}

To (72), (73), (76), (78) we must add, as usual, the Maxwell equations (63) in the region external to $\Omega, \boldsymbol{H} \rightarrow \boldsymbol{H}_{\text {ext }}$ at infinity, and continuity of $\boldsymbol{H}$ across $\partial \Omega$. In addition we also need to consider the need for a boundary condition on (76). This equation provides the system with one real characteristic, so that we must give a boundary condition on it wherever the characteristic points into the domain, i.e. whenever $\boldsymbol{v} \cdot \boldsymbol{n}<0$, where $\boldsymbol{n}$ is the outward normal to $\partial \Omega$. Physically this is saying that whenever vorticity is moving out of the sample we must not impose any condition, but whenever it is moving into the sample we must give the flux of vorticity through the boundary. This is similar to the Euler equations for an inviscid fluid, where the tangential component of vorticity must be given at any inflow boundary. Note that the region of the boundary over which $\boldsymbol{v} \cdot \boldsymbol{n}<0$ is unknown a priori, but depends on the solution of the equations. Thus the region over which a boundary condition must be applied changes as the solution evolves. This makes the mathematical analysis of the system (72), (73), (76), (78) formidable.

When $\boldsymbol{v} \cdot \boldsymbol{n}<0$ we must specify the flux of vorticity through the boundary, i.e. we must specify $(\boldsymbol{\omega} \wedge \boldsymbol{v}) \wedge \boldsymbol{n}$. We have seen that the analysis of the helical instability of individual vortices in [55] shows that the instability grows in such a way as to try to make the vortex normal to the driving current. This suggests that when new vortices are created they should be such that $\boldsymbol{\omega}$ is perpendicular to $\boldsymbol{J}$. Then, with the mean-field velocity law (80) we have

$$
\boldsymbol{\omega} \wedge \boldsymbol{v}=|\boldsymbol{\omega}| \boldsymbol{J}
$$

which is perpendicular to $\boldsymbol{n}$. Hence the natural conditions to give on an inflow boundary are $\boldsymbol{\omega} \cdot \boldsymbol{J}=0$ and $|\boldsymbol{\omega}|$ specified.

To determine the value of $|\boldsymbol{\omega}|$ we must return to the Ginzburg-Landau model which will describe the details of the generation of vortices. An analysis in [14] shows that the screening current flowing 
near the surface of a superconductor becomes unstable when it reaches a certain magnitude, $J_{\text {nucl }}$ say, and suggests that this is what leads to the creation of vortices near the boundary. Thus it is natural to set $|\boldsymbol{\omega}|=0$ if $|\boldsymbol{J}|<J_{\text {nucl }}$. For a smooth boundary, which leads to the maximum value of $J_{\text {nucl }}$, the calculation in [14] gives $J_{\text {nucl }}=2 \kappa \nu^{2} / \sqrt{27}$ in these units. In this case, since we have the restriction $\nu \gg \kappa^{-1 / 2}$ in the present model (corresponding to a dimensional magnetic field $\ll H_{c}$ ), $J_{\text {nucl }} \gg 1$ and vortices will not be generated at the boundary for this range of magnetic fields. However, as shown in [57], $J_{\text {nucl }}$ may be an order of magnitude smaller for boundaries with small asperities (and may even be nonconstant around the boundary), so even in the present model it is sensible to suppose that vortices are nucleated once the magnitude of $\boldsymbol{J}$ reaches a critical value.

For $|\boldsymbol{J}|>J_{\text {nucl }}$ one possibility is to suppose that there is a local equilibrium near the boundary so that $|\boldsymbol{\omega}|=|\boldsymbol{H}|$. Alternatively, one may suppose that the timescale for vortex generation is much smaller than that for vortex motion, so that vortices are generated rapidly until the magnitude of the current is reduced below the nucleation value. In this case $|\boldsymbol{\omega}|$ would not be given, but would be determined from the constraint that $|\boldsymbol{J}| \leq J_{\text {nucl }}$ on the boundary. Which of these conditions leads to a well-posed problem mathematically is still an open problem. The determination of the correct boundary conditions to apply to the vortex-density model is one area where numerical solution of the full Ginzburg-Landau problem could provide some useful clues.

\subsection{Two dimensions}

The equations (72), (73), (76), (78), (63) and boundary conditions become much simpler in two dimensions. With $\boldsymbol{H}=(0,0, H(x, y, t)), \boldsymbol{\omega}=(0,0, \omega(x, y, t))$ we have

$$
\begin{aligned}
-\nabla^{2} H+H & =\omega, \\
\frac{\partial \omega}{\partial t}+\operatorname{div}(\omega \boldsymbol{v}) & =0, \\
\boldsymbol{v} & =-\operatorname{sign}(\omega) \nabla H .
\end{aligned}
$$

Note that since the vortices are all rectilinear there is no self-induced term in the law of motion, and since the current lies in the $x y$-plane there is no problem with the helical instability. As usual the boundary condition $H=H_{e x t}$ is applied on $\partial D$. As in three dimensions, whenever $\boldsymbol{v} \cdot \boldsymbol{n}<0$ we need to apply a boundary condition on $\omega$. It is natural to impose $\omega=0$ whenever $|\boldsymbol{J}|\left\langle J_{\text {nucl }}\right.$ and give for example $w=H_{\text {ext }}$ when $|\boldsymbol{J}| \geq J_{\text {nucl }}$. Alternatively, as in three dimensions, $\omega$ may be determined from the constraint $|\boldsymbol{J}| \leq J_{\text {nucl }}$ on the boundary.

The model (93)-(95) was first studied in [21]. In particular [21] examined steady-state solutions satisfying $\omega \boldsymbol{v}=\mathbf{0}$. Then either there are no vortices and $\omega=0$, or $\boldsymbol{v}=\mathbf{0}$ so that $H=\omega=$ constant. Thus the domain $D$ is divided into regions of constant vorticity $D_{i}, i=1, \ldots, n$, separated by regions of zero vorticity, and $H$ satisfies the following free-boundary problem

$$
\begin{aligned}
-\nabla^{2} H+H & =0 \quad \text { in } D \backslash \cup_{i=1}^{n} D_{i}, \\
H & =H_{\text {ext }} \quad \text { on } \partial D, \\
H & =H_{i} \quad \text { on } \partial D_{i}, \quad i=1, \cdots, n \\
\frac{\partial H}{\partial n} & =0 \quad \text { on } \partial D_{i} .
\end{aligned}
$$

It is shown in [21] that for $H_{1}$ less than and close enough to $H_{\text {ext }}$ there is only one patch of vorticity $D_{1}$, and the solution to (96)-(99) exists and is unique. In fact a solution with $H_{i}=H_{1}$ for all $i$ should exist for all $H_{1} \in\left(H^{*}, H_{e x t}\right]$, where $H^{*}$ is the minimum value of $H$ satisfying (96) on $D$ with $(97)$ on $\partial D$. 
In $[64,62],(93)-(95)$ are considered for the case $J_{n u c l}=\infty$, so that there is no nucleation on the boundary, and the existence of a weak solution which converges to a solution of (96)-(99) is proved.

Recently Sandier and Serfaty [60] have proved rigorously the convergence of minimisers of the Ginzburg-Landau free energy to solutions of (96)-(99) as $\kappa \rightarrow \infty$ for applied magnetic fields of order $(\log \kappa) / \kappa$, with $H_{i}$ given by $H_{\text {ext }}-(\log \kappa) / 2 \kappa$ for each $i$ (in the original scaling of magnetic field). If $H_{e x t}-(\log \kappa) / 2 \kappa<H^{*}$ (in particular, if $\left.H_{e x t}<(\log \kappa) / 2 \kappa\right)$ as $\kappa \rightarrow \infty$ the minimising solution is the Meissner solution with no vortices [58], while if $H_{\text {ext }}$ is much greater than $(\log \kappa) / \kappa$ the minimising solution has $D_{1}=D, H_{1}=\omega=H_{\text {ext }}$ so that the domain is full of vortices [59]. In these cases, and in the case that $H_{\text {ext }}=O((\log \kappa) / \kappa)$ but $H_{i} \neq(\log \kappa) / 2 \kappa$, the solutions of (96)-(99) correspond to the limit of local minimisers of the Ginzburg-Landau free energy, rather than the global minimiser. The "surface barrier" to vortex nucleation discussed above, whereby vortices are not nucleated until the surface current reaches a critical value, is the reason for this large number of local minima. In particular, it is shown in [10] that the Meissner solution remains a local minimiser even for magnetic fields of order one as $\kappa \rightarrow \infty$.

Finally we note that the alternative two-dimensional situation of a cylinder in a transverse magnetic field has been studied in [61, 33, 57]. In particular, in [33] the existence of a weak solution is proved and it is shown rigorously that in the limit $\epsilon \rightarrow 0$ the self-induced term may be neglected. Solutions comprising regions of non-zero vorticity separated by regions of zero vorticity also exist in this setting, and these have been studied in [61, 33, 57], including some partial results on the regularity of the free boundary.

\subsection{The long-wave limit}

Throughout we have been working on the lengthscale of the penetration depth, $\lambda_{D}$. In dimensional terms this is typically about $0.1 \mu \mathrm{m}$. Hence a typical superconducting sample will be very large on this scale. If we instead scale lengths with the sample size, $L_{D}$ say, and rescale time with $L_{D}^{2} / \lambda_{D}^{2}$ also, then we find

$$
\begin{aligned}
& \lambda^{2}(\operatorname{curl})^{2} \boldsymbol{H}+\boldsymbol{H}=\boldsymbol{\omega}, \\
& \operatorname{div} \boldsymbol{H}=0 . \\
& \frac{\partial \boldsymbol{\omega}}{\partial t}+\operatorname{curl}(\boldsymbol{\omega} \wedge \boldsymbol{v})=\mathbf{0}, \\
& \boldsymbol{v}=\operatorname{curl} \boldsymbol{H} \wedge \hat{\boldsymbol{\omega}}+\epsilon \operatorname{curl} \hat{\boldsymbol{\omega}} \wedge \hat{\boldsymbol{\omega}},
\end{aligned}
$$

where $\lambda=\lambda_{D} / L_{D}$ is the ratio of the penetration depth to the size of the sample. Now since the limit $\lambda \rightarrow 0$ is a singular one we expect there to be boundary layers near $\partial \Omega$ of thickness $\lambda$, which we will examine shortly. However, assuming that there are no rapid oscillations in $\boldsymbol{H}$ in the interior of the domain when $\lambda \ll 1$ we find to leading order that

$$
\begin{aligned}
\boldsymbol{H} & =\boldsymbol{\omega}, \\
\frac{\partial \boldsymbol{H}}{\partial t}+\operatorname{curl}(\boldsymbol{H} \wedge \boldsymbol{v}) & =\mathbf{0}, \\
\boldsymbol{v} & =\operatorname{curl} \boldsymbol{H} \wedge \hat{\boldsymbol{H}}+\epsilon \operatorname{curl} \hat{\boldsymbol{H}} \wedge \hat{\boldsymbol{H}},
\end{aligned}
$$

where $\hat{\boldsymbol{H}}$ is the unit vector in the direction of $\boldsymbol{H}$. It is not clear whether or not the model (105)(106) is ill-posed if the self-induced term is neglected, since it is not possible to give an infinitesimal perturbation to $\boldsymbol{H}$ without also perturbing $\boldsymbol{J}$, but it appears that may it exhibit the same instability 
as (78). Thus when we set $\epsilon$ to zero we must restrict ourselves to configurations in which $\boldsymbol{H} \cdot \boldsymbol{J} \equiv 0$ (i.e. $\boldsymbol{H}$ must have zero helicity).

The model (105)-(106) (with $\epsilon=0$ ) was written down by Brandt [12], based on the "Lorentz force" law $\boldsymbol{v}=\boldsymbol{J} \wedge \hat{\boldsymbol{H}}$ and the observation that for a moving magnetic field $\boldsymbol{E}=\boldsymbol{H} \wedge \boldsymbol{v}$; then, as we have seen, (105) is simply Faraday's law.

As mentioned earlier, (105), (106) can be derived directly from a multiple scale analysis of the Ginzburg-Landau equations [57], and have a greater range of validity than that of (72), (73), (76), (78), which are restricted to magnetic fields in the range $1 / \kappa \ll|\boldsymbol{H}| \ll 1$ in the original scaling of (11)-(12), corresponding to vortex separations $\kappa^{-1 / 2} \ll \nu \ll 1$. The restriction $|\boldsymbol{H}| \ll 1$ arises from the restriction $|\boldsymbol{J}| \ll 1$ when $\boldsymbol{H}$ varies on an order-one lengthscale, the latter restriction being necessary so that $f \approx 1$ in regions away from vortex cores. However, it is possible to have larger magnetic fields without having a larger electric current density, providing the magnetic field varies on a longer lengthscale. If the magnetic field varies on the lengthscale $L$ say (by comparison to the penetration depth, so, for example, we may expect $\left.L=L_{D} / \lambda_{D}\right)$, then $|\boldsymbol{J}| \sim|\boldsymbol{H}| / L \sim 1 /\left(L \kappa \nu^{2}\right)$. Hence the model will be valid providing

$$
\frac{1}{L} \ll \kappa \nu^{2} .
$$

This allows us to extend the range of magnetic fields to $1 / \kappa \ll|\boldsymbol{H}| \ll \kappa$, corresponding to vortex separations $\kappa^{-1} \ll \nu \ll 1$. The lower limit now is determined by the vortex core radius $1 / \kappa$. As this limit is approached the cores of individual vortices start to overlap and we are in the vicinity of $H_{c_{2}}$, the upper critical field.

However, when we allow this increase in magnetic field while not increasing the current density, we are weakening the dominance of the mean-field or average current over the local current due to the immediate neighbours of a particular vortex. The local perturbation to the current due to neighbouring vortices is $O(1 /(\kappa \nu))$ (from (57)), while the mean-field current is $O\left(1 /\left(L \kappa \nu^{2}\right)\right.$ ). Hence the mean-field will dominate, and the dynamics will be determined by the average current density, only if $1 / L \gg \nu$, corresponding to $|\boldsymbol{J}| \gg \sqrt{|\boldsymbol{H}| / \kappa}$. In the alternative case $1 / L \ll \nu$ then the dominant forces on a vortex are local. In this case the vortices will form a strong lattice even under strong forcing, and the bulk motion of vortices is likely to be through the motion of dislocations in the lattice. The dependence of the bulk properties of the solution on the details of the local behaviour makes such a situation more difficult to model, and we do not consider it further here. In fact the problem is analogous to deriving macroscopic models of two-dimensional plastic flow based on local models of elastic crystal lattices. We note though that it would be interesting to try and relate the condition $1 / L \approx \nu$ to the widely reported phenomenon of vortex lattice melting, since this switch from local to global forces dominating would seem to coincide with the switch from a vortex lattice to a vortex liquid.

In summary, the range of validity of the model (105)-(106) is

$$
\nu \ll \frac{1}{L} \ll \kappa \nu^{2},
$$

where $L$ is the lengthscale for the variation of the magnetic field and $\nu$ is the vortex separation (both by comparison to the penetration depth, $\lambda_{D}$ ). This corresponds to magnetic fields

$$
1 / \kappa \ll|\boldsymbol{H}| \ll \kappa
$$

and electric currents

$$
\sqrt{\frac{|\boldsymbol{H}|}{\kappa}} \ll|\boldsymbol{J}| \ll 1
$$


Note that if the magnetic field varies over a lengthscale comparable to the sample size (as we might expect) so that $L=L_{D} / \lambda_{D}$, then this limits the vortex liquid models to samples of size $L_{D} \ll \kappa \lambda_{D}$. The ranges of validity of both the finite $\lambda$ vortex-density model and the long-wave approximation $\lambda \rightarrow 0$ are shown schematically in Figure 5 .

Returning to the long-wave limit of (72), (73), (76), (78), we note again that the limit $\lambda \rightarrow 0$ is a singular perturbation and we must be aware of the possibility of boundary layers on the edge of the sample when we impose boundary conditions on (105), (106). Since equations (105), (106) form a vector nonlinear diffusion equation for $\boldsymbol{H}$, if there were no boundary layer the natural condition would be to impose continuity of $\boldsymbol{H}$ on the boundary, as well as the usual Maxwell equations (63) outside $\Omega$ and the condition at infinity. Note that even though the singular perturbation is in (72), in the limit the boundary condition on $\boldsymbol{H}$ is retained, while the difficult boundary condition on $\boldsymbol{\omega}$ is lost. Before we discuss the possibility of boundary layers, let us first examine the simpler two-dimensional situation.

\subsection{The long-wave limit in two dimensions}

With the usual two-dimensional setting we have

$$
\begin{aligned}
\omega & =H, \\
\frac{\partial H}{\partial t} & =\operatorname{div}(|H| \nabla H) .
\end{aligned}
$$

This is a version of the well-known porous medium equation (see [3] for example). Note though that unlike the pressure in the porous medium equation, the magnetic field $H$ can take both positive and negative values.

The natural boundary condition to give on (112) is to specify $H$ on the boundary. However, a local analysis in the vicinity of the boundary gives the possibility of a vortex-free region near the boundary of thickness $\lambda$, as described in $\S 4.3$. If the domain is sufficiently smooth that the radius of curvature of the boundary is much bigger than $\lambda$ then locally the boundary is flat and the domain a half-space. The boundary-layer problem is then

$$
\begin{aligned}
-H_{x x}+H & =0 \quad \text { for } 0<x<d, \\
H(0) & =H_{\text {ext }}, \\
H_{x}(d) & =0
\end{aligned}
$$

where $x$ is the coordinate normal to the boundary (rescaled with $\lambda$ ), and $d$ is the thickness of the vortex-free region. The solution is

$$
H=\frac{H_{0} \cosh (d-x)}{\cosh d} .
$$

Now, the boundary condition which should be applied on the long-wave model (112) is the value of the magnetic field at the edge of the vortex-free zone, namely $H(d)$. To determine this we still need to determine $d$. To do this we note that the current density at the surface of the material is

$$
J(0)=\frac{H_{0} \sinh d}{\cosh d},
$$

and we have the constraint that this must be less than $J_{\text {nucl }}$ if we are not to be generating more vortices. Now, if the magnetic field is being raised then no new vortices will be created until 


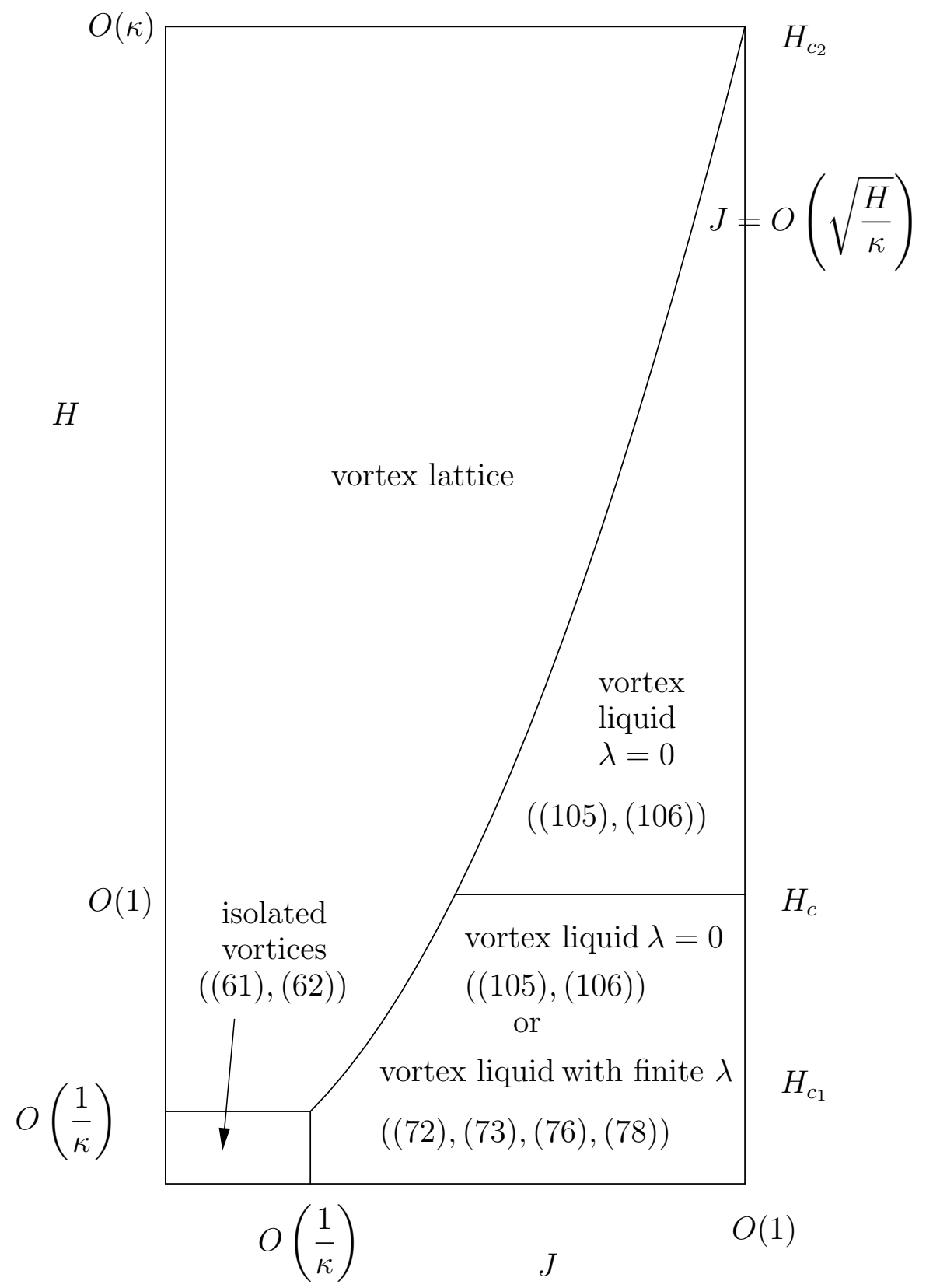

Figure 5: Schematic diagram of the region of validity of the various models. 
$J(0)=J_{n u c l}$. Once they are created they will move into the sample until the magnetic field inside has increased sufficiently to bring $J(0)$ back down to $J_{\text {nucl }}$ on the boundary. Hence for increasing magnetic fields $J(0)=J_{\text {nucl }}$ and $H(d)=\left(H_{\text {ext }}^{2}-J_{\text {nucl }}^{2}\right)^{1 / 2}$. For decreasing magnetic fields, since $H(d) \leq H_{\text {ext }}$, we have $H(d)=H_{\text {ext }}$. Hence there is hysteresis in the boundary condition, which we may write succinctly as

$$
H(d) \in\left[\left(H_{\text {ext }}^{2}-J_{\text {nucl }}^{2}\right)^{1 / 2}, H_{\text {ext }}\right] .
$$

Note that $H(d) \neq H_{\text {ext }}$ corresponds to a surface current sheet in the outer model, which is the screening current flowing in the vortex-free region. Such a surface current was also included in the model by Brandt [12]. Note also that in cases where $J_{n u c l} \ll H_{\text {ext }}$ there is no hysteresis, which in particular happens whenever $H_{\text {ext }} \gg 1$ in the original scaling of (11)-(12), since the maximum value of $J_{\text {nucl }}$ in that scaling is $O(1)$.

So far we have been using the linear version of the equation for the magnetic field, under the assumption that $H_{\text {ext }}$ and $J_{\text {nucl }}$ are much less than one (i.e. the surface is rough). If the surface is polished so that $J_{n u c l}$ is order one, and the magnetic field is also order one, then the boundary layer is described by the nonlinear Ginzburg-Landau equations, giving

$$
\begin{aligned}
Q_{x x} & =Q-Q^{3} \quad \text { for } 0<x<d, \\
Q_{x}(0) & =H_{\text {ext }}, \\
Q(d) & =0
\end{aligned}
$$

where $Q$ is the tangential component of $\boldsymbol{Q}$ (whose normal component is zero to leading order), and we are in the original scaling (11)-(12) . However, in this case we still have the first integral

$$
\left(Q_{x}\right)^{2}=Q^{2}-\frac{Q^{4}}{2}+H(d)^{2},
$$

so that

$$
H(d)=\left(H_{e x t}^{2}-Q(0)^{2}+\frac{Q(0)^{4}}{2}\right)^{1 / 2} .
$$

It is shown in [14] that this solution becomes unstable when $Q(0) \geq 1 / \sqrt{3}$, which is conjectured to be the onset of vortex nucleation. Since the maximum value of $Q$ is on the boundary this gives

$$
H(d)=\left(H_{e x t}^{2}-H_{n u c l}^{2}\right)^{1 / 2},
$$

where $H_{\text {nucl }}$ is the magnetic field required to nucleate vortices in an initially empty half-space, which is $\sqrt{5 / 18}$ in this scaling. Thus the boundary condition is of the same form as in the linear case.

From this two-dimensional boundary layer analysis we can conclude that there will be no boundary layer when $H_{\text {ext }} \gg J_{\text {nucl }}$, so that in three dimensions also in this case we will simply impose continuity of the magnetic field.

\subsection{Pinning in vortex-density models}

The effect of the introduction of pinning into the vortex-density models depends upon the lengthscale for variations in the pinning potential by comparison to the separation of vortices.

Let us first assume that the vortex separation is much smaller than the typical lengthscale for variations in pinning potential. Then the pinning potential will be slowly varying on the

\footnotetext{
${ }^{4}$ The nonlinear version of (96)-(99) is studied in [11].
} 
$\eta$ lengthscale and the averaging process will proceed as before, leading to the modified London equations:

$$
\begin{aligned}
\operatorname{curl}\left(\frac{1}{a(\boldsymbol{x})} \operatorname{curl} \boldsymbol{H}\right)+\boldsymbol{H} & =\boldsymbol{\omega} \\
\operatorname{div} \boldsymbol{H} & =0 .
\end{aligned}
$$

The law of vortex conservation is unchanged:

$$
\frac{\partial \boldsymbol{\omega}}{\partial t}+\operatorname{curl}(\boldsymbol{\omega} \wedge \boldsymbol{v})=\mathbf{0},
$$

but the law of motion is modified to include the effects of the pinning potential:

$$
\boldsymbol{v}=\frac{\boldsymbol{J}}{a(\boldsymbol{x})} \wedge \hat{\boldsymbol{\omega}}+\epsilon \operatorname{curl} \hat{\boldsymbol{\omega}} \wedge \hat{\boldsymbol{\omega}}-\epsilon \log a
$$

However, we see that the pinning potential is weak, by comparison to the force exerted on a vortex by all the other vortices, when $\epsilon=\left(\nu^{2} \log \kappa\right) / 2 \ll 1$.

The other limit we can consider is that the variation in the pinning potential occurs on a lengthscale $\delta$ which is much shorter than the separation of the vortices. This leads to so-called critical-state models, which we will describe in Section 6.

First, in Section 5, we examine the possibility of modifying the vortex density models to capture the large-scale effects of the small-scale helical instability of individual vortices.

\section{$5 \quad$ Vortex density models including the helical instability}

As we have said, the model (72), (73), (76), (80) (i.e. with $\epsilon=0$ ) is good so long as $\boldsymbol{\omega} \cdot \boldsymbol{J} \equiv 0$. If there is no symmetry to guarantee $\boldsymbol{\omega} \cdot \boldsymbol{J} \equiv 0$ then we may regularise the model by retaining a finite $\epsilon$, thus capturing the instability and preventing an infinite growth rate, or we may try and model the large-scale effects of the small-scale instability on the average fields $\boldsymbol{H}, \boldsymbol{J}$ etc. We will attempt the latter in this section, confining the regularisation of the instability to the small scale. Brandt [12] does this by decomposing the electric current density into components parallel and perpendicular to the magnetic field, and supposing that each gives rise to a different resitivity, the former due to the helical instability and "vortex cutting", the latter due to vortex translation. Thus he writes

$$
\boldsymbol{E}=\rho_{\|} \boldsymbol{J}_{\|}+\rho_{\perp} \boldsymbol{J}_{\perp}
$$

with $\rho_{\|}=\rho_{\|}\left(\boldsymbol{H}, \boldsymbol{J}_{\|}\right), \rho_{\perp}=\rho_{\perp}\left(\boldsymbol{H}, \boldsymbol{J}_{\perp}\right)$, although he is primarily interested in the variation of the $\rho$ 's with temperature, which will not concern us here. Chapman \& Richardson [20] consider in more detail the implications of the evolution of the helical instability, and arrive at a slightly different conclusion. We follow their argument here.

Let us first recall that the helical instability for a single vortex has a most unstable wavenumber of $|\boldsymbol{J} \cdot \hat{\boldsymbol{\omega}}| /(2 \epsilon)$ which has a growth rate of $|\boldsymbol{J} \cdot \hat{\boldsymbol{\omega}}|^{2} /(4 \epsilon)$, and that the nonlinear evolution of the helix is such as to try to make the vortex perpendicular to the current (see Figure 4). Now suppose that all neighbouring vortices are undergoing the same instability. Eventually they will grow large enough to reconnect with each other. Note that the wavelength of the instability is much smaller than the vortex separation in the liquid regime we are considering, so that most of the evolution is in the nonlinear regime, and in fact the curvature term quickly becomes unimportant in the evolution of the helix. Modelling the evolution of the average vorticity is difficult under these circumstances. 
Clearly if the current density and vorticity are both uniform the average vorticity remains constant. But how should it evolve when the current density is non-uniform, so that the pitch of each helix is not the same? A solution to this problem comes from the observation made earlier that the law of conservation of vorticity is equivalent to Faraday's law. During the evolution of the helical instability, an electric field is generated which is parallel to the local current density, and which is therefore much easier to average. The magnitude of this electric current is proportional to the vorticity, to the component of the current density in the direction of the vorticity, and to the current density itself. To see this let us treat the helix as a superposition of the original straight vortex and a set of expanding vortex rings, as shown in Figure $6^{5}$. The number of vortices per unit area is $|\boldsymbol{\omega}|$, the number of rings per unit length on each vortex is $|\boldsymbol{J} \cdot \hat{\boldsymbol{\omega}}| /(4 \pi \epsilon)$, and the rate of expansion of the rings once they are sufficiently large that the self-induced restoring term is negligible (i.e. their radius $\gg 1 / \epsilon)$ is $|\boldsymbol{J}|$. However, the local average of the electric field generated by the expanding rings is also proportional to the length of each ring, which changes in time as the instability evolves, but will be of the order of the separation between vortices $\nu$, since the rings will stop growing when they reconnect with neighbouring vortices.

If we perform a local average in time also and include this extra component of the electric field due to the evolving small scale instability with our original two components due to vortex translation and superconducting electron acceleration, we have

$$
\boldsymbol{E}=\operatorname{curl} \boldsymbol{H}_{t}+\boldsymbol{\omega} \wedge(\boldsymbol{J} \wedge \hat{\boldsymbol{\omega}})+\frac{\alpha \nu}{\epsilon}|\boldsymbol{J} \cdot \boldsymbol{\omega}| \boldsymbol{J},
$$

where $\alpha$ is an order-one parameter. Faraday's law then gives the new law of vortex conservation as

$$
\boldsymbol{\omega}_{t}+\operatorname{curl}(\boldsymbol{\omega} \wedge(\boldsymbol{J} \wedge \hat{\boldsymbol{\omega}}))+\frac{\alpha \nu}{\epsilon} \operatorname{curl}(|\boldsymbol{J} \cdot \boldsymbol{\omega}| \boldsymbol{J})=0 .
$$

The factor of $\nu / \epsilon \sim 1 / \nu \log \kappa$ in the new term here means that in many cases it dominates the original terms in (130), and we can consider the implications of the limit $\nu / \epsilon \rightarrow \infty$. Before we do this let us first try and determine what the new term means in our original vortex-conservation argument.

Consider again the open surface $S$ and the vortices which intersect it, and allow both to be convected with the velocity field $\boldsymbol{v}$ while at the same time allowing the vortices to develop the helical instability. To simplify the picture we again treat the vortices as locally straight plus a stack of vortex rings. Then there will be a new contribution to the number of vortices which cross $S$ due to the vortex rings. The rings which are generated in the interior of $S$ will each intersect $S$ twice, once in the positive sense and once in the negative sense, so that they will not contribute to the total. However, the rings which are generated on the boundary of $S$ will only intersect $S$ once (see Figure 7). Now, if the density of rings around the boundary is constant then these will also add up to zero, but if there are more in some regions than others they will generate an additional nonzero contribution to the total. From (130) we find

$$
\begin{aligned}
\frac{d}{d t} \int_{S} \boldsymbol{\omega} \cdot \boldsymbol{d} \boldsymbol{S} & =-\frac{\alpha \nu}{\epsilon} \int_{S} \operatorname{curl}(|\boldsymbol{J} \cdot \boldsymbol{\omega}| \boldsymbol{J}) \cdot \boldsymbol{d} \boldsymbol{S} \\
& =-\frac{\alpha \nu}{\epsilon} \int_{\partial S}|\boldsymbol{J} \cdot \boldsymbol{\omega}| \boldsymbol{J} \cdot \boldsymbol{d} \boldsymbol{s} .
\end{aligned}
$$

The source term on the right-hand side here is a measure of the number of vortex rings generated around the boundary $\partial S$.

\footnotetext{
${ }^{5}$ There is an interesting analogy here between the vortices generated by this helical instability and a Frank-Reed source of dislocations in an elastic crystal, which generates circular dislocations in the middle of an elastic crystal.
} 


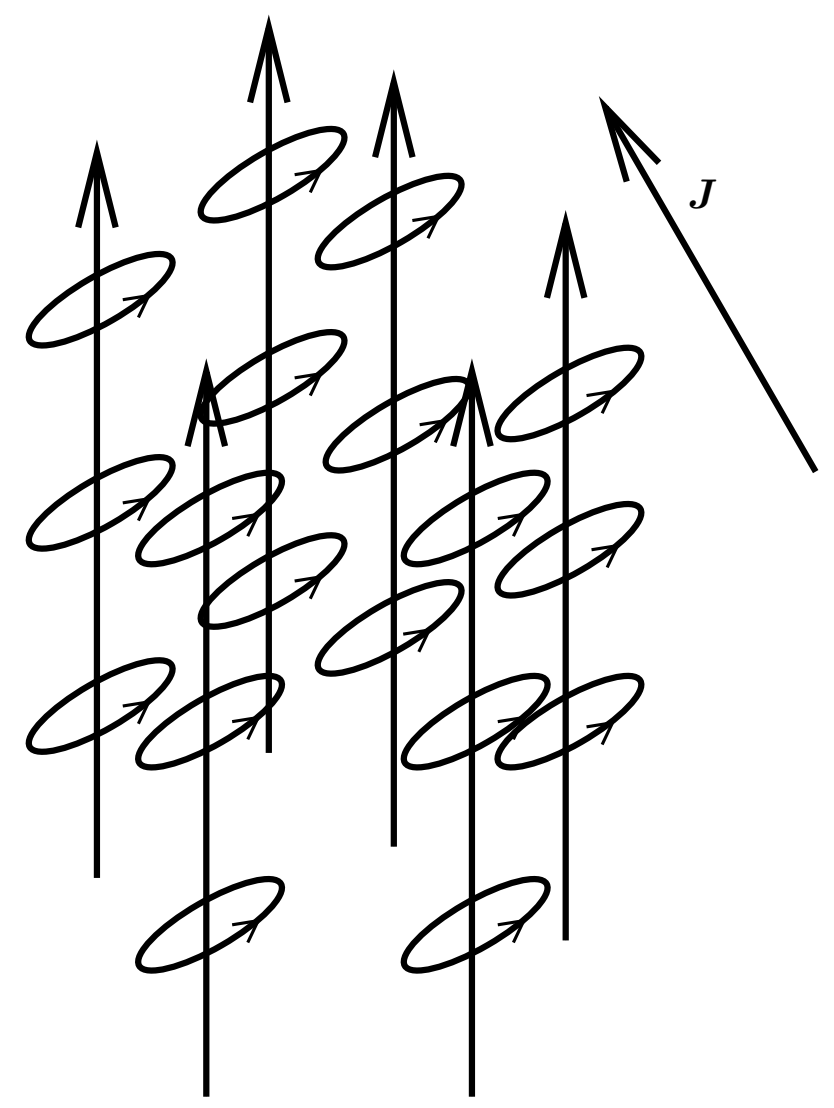

Figure 6: The vortex rings equivalent to the helical instability and the electric field they generate.

\subsection{The limit of the modified model as $\nu / \epsilon \rightarrow \infty$}

As noted above, since the new term we have added to the electric field is dominant over the old terms by a factor of $\nu / \epsilon$, we may consider the implications of the limit $\nu / \epsilon \rightarrow \infty$. If $\boldsymbol{\omega} \cdot \boldsymbol{J} \neq 0$ then the time derivative of the vorticity is very large, and we must rescale time onto a short timescale $t=\epsilon \bar{t} /(\alpha \nu)$. The evolution on this short timescale is governed by the equations

$$
\begin{aligned}
\boldsymbol{\omega}_{\bar{t}}+\operatorname{curl}(|\boldsymbol{\omega} \cdot \boldsymbol{J}| \boldsymbol{J}) & =\mathbf{0}, \\
\boldsymbol{J} & =\operatorname{curl} \boldsymbol{H}, \\
\operatorname{curl}^{2} \boldsymbol{H}+\boldsymbol{H} & =\boldsymbol{\omega}, \\
\operatorname{div} \boldsymbol{H} & =0 .
\end{aligned}
$$

If the boundary conditions are also varying on this short timescale then this is the only model which needs to be addressed. However, if the boundary conditions vary on the original timescale then they are constant during this short time relaxation, and it would seem natural (based on our local picture of what is happening in Figure 4) for the long-time behaviour of this short-time solution to lead to $\boldsymbol{\omega} \cdot \boldsymbol{J}=0$, although this is an open question mathematically. If this is the case, then the role of the short timescale is to rapidly adjust the solution so that the constraint $\boldsymbol{\omega} \cdot \boldsymbol{J}=0$ is always satisfied. The evolution of the solution under this constraint is then governed by the equations on the original timescale.

However, we must be careful not to simply set $\boldsymbol{\omega} \cdot \boldsymbol{J}=0$ in (129) to obtain these equations; at the next order in (129) the first-order correction to $\boldsymbol{\omega} \cdot \boldsymbol{J}$ will balance the remaining terms. Nevertheless, we can still gather some leading-order information from this equation by taking the 


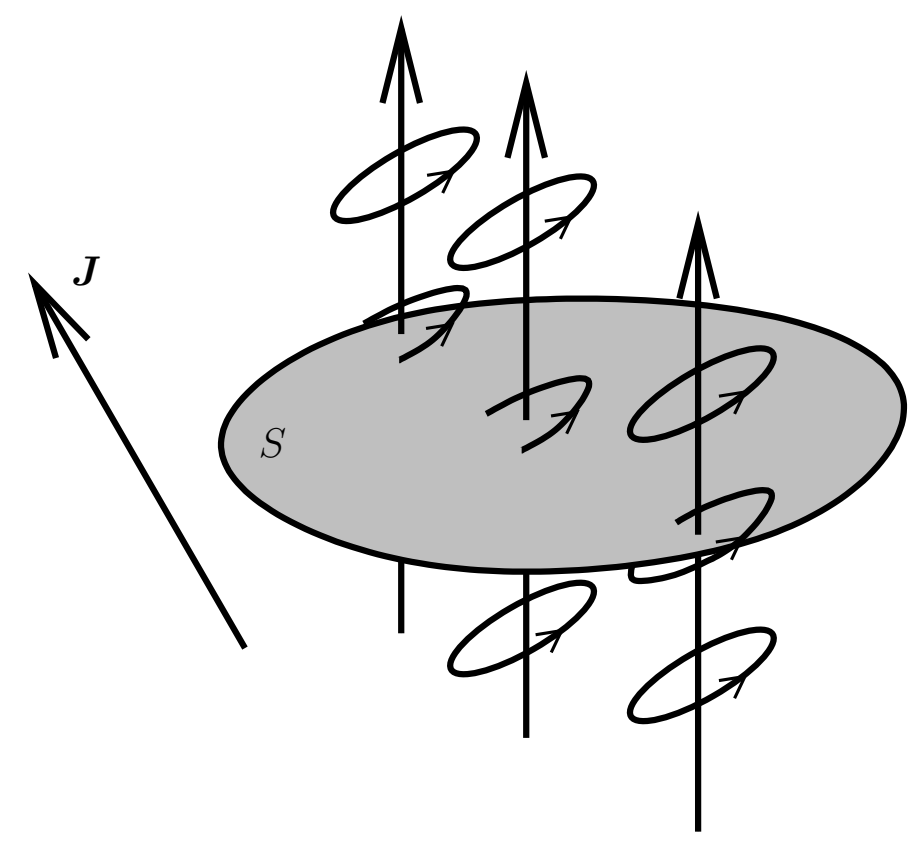

Figure 7: The evolution of the surface $S$ including the vortex rings generated by the instability.

cross product with $\boldsymbol{J}$ to eliminate the final term. This results in

$$
\boldsymbol{E}=\boldsymbol{J}_{t}+\varsigma \boldsymbol{J}
$$

where $\varsigma$ is unknown, and is determined from the constraint that $\boldsymbol{\omega} \cdot \boldsymbol{J}=0$.

Note that this model still contains the effects of the helical instability, even though we now have $\boldsymbol{\omega} \cdot \boldsymbol{J}=0$ to leading order: it is exactly because of the instability that $\varsigma$ is unknown. A curious feature of the three-dimensional model has emerged here: it does not reduce to the two-dimensional model in the case $\boldsymbol{\omega} \cdot \boldsymbol{J} \equiv 0$, since now the constraint is no constraint and $\varsigma$ is undetermined.

\subsection{The long-wave limit of the modified model}

In the long-wave limit, scaling length with $L_{D}$ and rescaling time with $L_{D}^{2} / \lambda_{D}^{2}$ as in Section 4.4 we have $\boldsymbol{H}=\boldsymbol{\omega}$ as before and

$$
\begin{aligned}
\operatorname{div} \boldsymbol{H} & =0, \\
\boldsymbol{H}_{t}+\operatorname{curl} \boldsymbol{E} & =\mathbf{0} \\
\boldsymbol{E} & =\boldsymbol{H} \wedge(\boldsymbol{J} \wedge \hat{\boldsymbol{H}})+\frac{\alpha \nu}{\epsilon}|\boldsymbol{J} \cdot \boldsymbol{H}| \boldsymbol{J} .
\end{aligned}
$$

Note that the extra component to the electric field in (138) is parallel to $\boldsymbol{J}$, rather than $\boldsymbol{J}_{\|}$as in the Brandt model [12]. This is because the helical instability evolves to make the vortex perpendicular to the current, and so, after the initial transient, produces an electric field parallel to $\boldsymbol{J}$ rather than $\boldsymbol{J}_{\|}$.

To (136)-(138) we must add as usual the Maxwell equations (63) in the region exterior to $\Omega$, the boundary condition at infinity, and the continuity of $\boldsymbol{H}$ across the interface. Note that (138) effectively replaces Ohm's law in the superconductor. 


\subsection{The long-wave limit of the modified model in the limit $\nu / \epsilon \rightarrow \infty$}

As before there are two timescales to consider. On the short timescale we must rescale $\boldsymbol{E}$ with $\alpha \nu / \epsilon$ and time with $\epsilon /(\alpha \nu)$ to give

$$
\begin{aligned}
\boldsymbol{E} & =|\boldsymbol{J} \cdot \boldsymbol{H}| \boldsymbol{J}, \\
\boldsymbol{J} & =\operatorname{curl} \boldsymbol{H}, \\
\operatorname{div} \boldsymbol{H} & =0 \\
\boldsymbol{H}_{\bar{t}}+\operatorname{curl} \boldsymbol{E} & =\mathbf{0} .
\end{aligned}
$$

Thus we have a material in which the conductivity is $|\boldsymbol{J} \cdot \boldsymbol{H}|$, which effectively gives a nonlinear diffusion equation for the magnetic field in which the diffusivity is the modulus of the helicity of $\boldsymbol{H}$.

If the boundary conditions are constant on this short timescale, and if the longtime behaviour of this model leads to $\boldsymbol{J} \cdot \boldsymbol{H}=0$, then on the long timescale we find

$$
\begin{aligned}
\boldsymbol{E} & =\varsigma \boldsymbol{J}, \\
\boldsymbol{J} & =\operatorname{curl} \boldsymbol{H}, \\
\operatorname{div} \boldsymbol{H} & =0, \\
\boldsymbol{H}_{t}+\operatorname{curl} \boldsymbol{E} & =0,
\end{aligned}
$$

with $\varsigma$ determined from the constraint that

$$
\boldsymbol{J} \cdot \boldsymbol{H}=0 .
$$

Note that a convenient way to impose the constraint of zero helicity on $\boldsymbol{H}$ is by the use of Clebsch potentials, the general solution of (147) being given by $\boldsymbol{H}=f \nabla g$ with $f$ and $g$ arbitrary scalar functions.

\section{Critical-state models}

Here we consider the limit in which the pinning potential is rapidly varying, and we aim to homogenise. If we look locally near a single vortex which is in the deepest local well and consider the effect of applying a current $\boldsymbol{J}$ to it, we see that if the current is not sufficient to cause the vortex to leave its local well, then it will move up the side of the well until the attraction from the potential balances the applied current (see Figure 8). In this case the vortex will have moved a distance of order $\delta$. Now, if we increase the current $\boldsymbol{J}$, then at some point it will be sufficient to cause the vortex to leave its local well and it will jump into the next well. However, since the vortex was in the deepest local well the current will also be sufficient to cause the vortex to leave the neighbouring well. It will continue in this way until it has moved an order-one distance, until either the local well depth has increased, or until the local current density has decreased sufficiently to catch the vortex. Hence, in the limit that $\delta \rightarrow 0$, we arrive at a stick-slip mobility law: if the local current density is less that a critical value, $J_{c}$ say (which may be a function of position), then the vortex does not move, while if the local current density is greater than than $J_{c}$ then the vortex will move, but at a reduced speed due to moving through the potential wells. If the distribution of well depth is nonuniform, so that there are a range of depths locally, then it is possible to see how the critical current in this stick-slip model may depend on $|\boldsymbol{\omega}|$. If we add more and more vortices locally we 


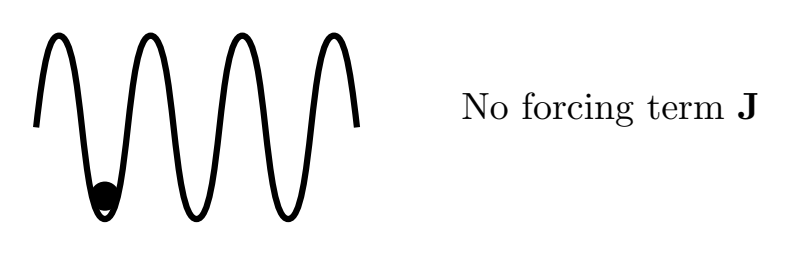
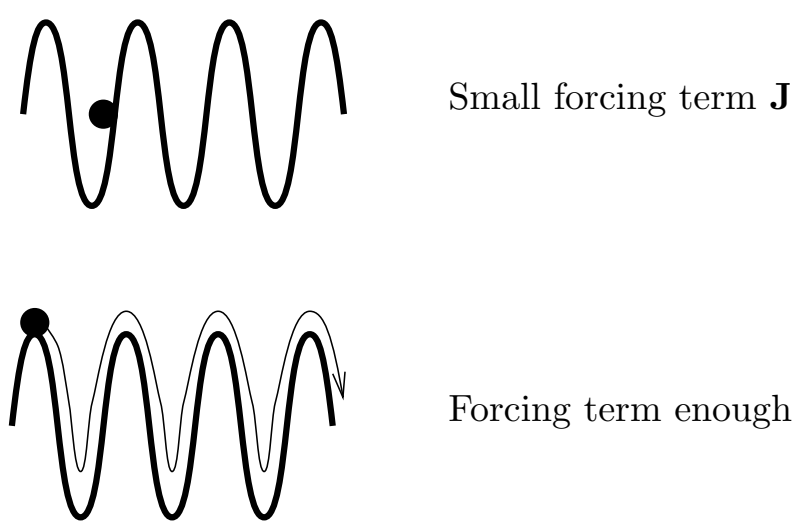

Forcing term enough to depin

Figure 8: Pinning of a single vortex by a rapidly varying pinning potential.

are having to make use of shallower and shallower wells, thus reducing the critical current required to start some of the vortices moving.

From (127) the critical current density in these scalings will be of order $\nu^{2} \delta^{-1} \log \kappa$. Note that the average speed of the vortex as it moves through the wells will be

$$
\bar{v}=\frac{\delta}{\int_{0}^{\delta} 1 / v d x}
$$

since $v$ is given as a function of position depending on the pinning potential.

We have described the interaction of a single vortex with a rapidly varying potential, which is the case $\delta \ll \nu$. A similar scenario exists in the complementary situation $\nu \ll \delta$ with the single vortex replaced by a "pool" of vorticity, as shown in Figure 9. Applying a forcing term now corresponds to "tilting" the potential, and again there will be a critical current at which the well can no longer hold the pool. In this case it is clear that the critical current will depend on the vortex density.

We have been describing the two-dimensional sitation in which the vortices are rectilinear and the pinning potential is also independent of $z$. In general in three dimensions the pinning sites will not be columnar and aligned with the vortices (although there have been attempts to try to introduce columnar defects into materials). The vortices will instead be pinned at certain places along their length, splaying out in between pinning sites as shown schematically in Figure 10. Now as well as the background driving current the self-induced curvature term comes into play in trying to drive the vortex out of the potential well. However, the result of homogenising the pinning potential should still be a stick/slip law.

Thus, after homogenising the pinning potential the law of motion (80) is modified to be

$$
\boldsymbol{v}=F(|\boldsymbol{J} \wedge \hat{\boldsymbol{\omega}}| ; \boldsymbol{x}, \boldsymbol{\omega}) \hat{\boldsymbol{J}} \wedge \hat{\boldsymbol{\omega}}
$$



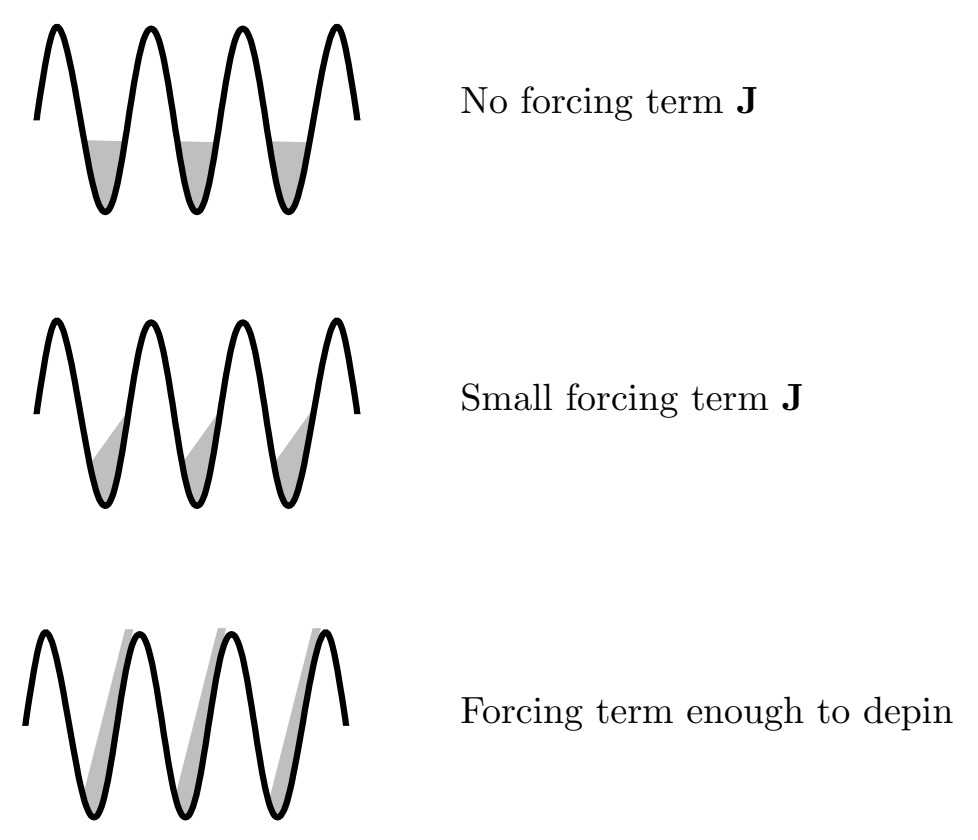

Figure 9: Pinning of a vortex pool by a rapidly varying pinning potential.

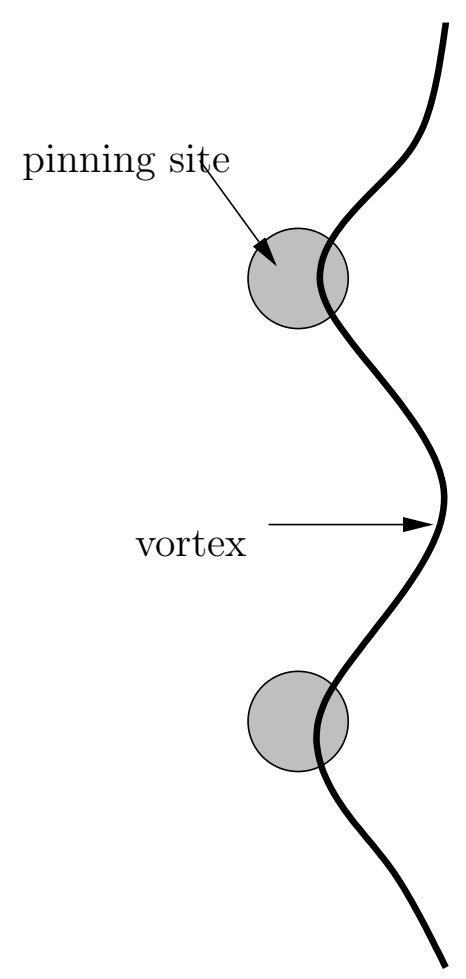

Figure 10: Schematic diagram of a vortex line being pinned in three dimensions. 
where $\hat{\boldsymbol{J}}$ is the unit vector in the direction of $\boldsymbol{J}$, and $F(J)=0$, for $J<J_{c}(\boldsymbol{x}, \boldsymbol{\omega})$ (see Figure 11). Note also that (124) becomes

$$
\operatorname{curl}\left(\frac{1}{\hat{a}(\boldsymbol{x})} \operatorname{curl} \boldsymbol{H}\right)+\boldsymbol{H}=\boldsymbol{\omega},
$$

where $\hat{a}$ is the effective equilibrium density of superconducting electrons, which is not simply the local average of $a$, but must be determined through a multiple scales analysis. Overall, we are confronted with an interesting "double" homogenisation problem, of both the pinning potential and the vortex density. Given $\boldsymbol{\omega}$, the determination of $\hat{a}$ is a standard problem in homogenisation; determining the law of motion of vortices is not. We have approached the problem one step at a time for ease of exposition, first homogenising vortices and then homogenising the pinning potential; the canonical problem is really a multiple scales analysis of the Ginzburg-Landau equations in which the vortex separation $\nu$, and $\delta$, the lengthscale for variations in $a$, both tend to zero in such a way that the critical current is order one. We are not aware of any literature that considers homogenisation in this canonical limit.

Returning to (149)-(150), the simplest model of this form will have $J_{c}$ constant, $\hat{a}=1$, and $F$ not explicitly dependent on $\boldsymbol{x}$ or $\boldsymbol{\omega}$. However, even with strong pinning such as this, the law of motion (149) still has the possibility of instability with unbounded growth, since if a large current is applied parallel to $\boldsymbol{\omega}$ an infinitesimal perturbation can take $|\boldsymbol{J} \wedge \hat{\boldsymbol{\omega}}|$ above $J_{c}$. Therefore, as in $\S 4$, such a model is restricted to situations in which $\boldsymbol{\omega} \cdot \boldsymbol{J} \equiv 0$. In the general case, if we model the effects of the instability rather than regularising it through a self-induced curvature term, as in Section 5, we find that there should be two critical currents, one "perpendicular" critical current $J_{c \perp}$ which is the threshold for vortex translation, and one "parallel" critical current $J_{c \|}$ which determines whether the helical instability can develop. The relative size of these will depend on the nature and distribution of the pinning sites. Thus we find

$$
\boldsymbol{E}=\boldsymbol{J}_{t}+\boldsymbol{\omega} \wedge \boldsymbol{v}+G(|\boldsymbol{J} \cdot \hat{\boldsymbol{\omega}}|,|\boldsymbol{J}|)|\boldsymbol{\omega}| \hat{\boldsymbol{J}},
$$

where vortex conservation is given by Faraday's law (16), and

$$
G(|\boldsymbol{J} \cdot \hat{\boldsymbol{\omega}}|,|\boldsymbol{J}|)=0 \text { if }|\boldsymbol{J} \cdot \hat{\boldsymbol{\omega}}|<J_{c \|} \cdot
$$

Again, we have Maxwell's equations (63) in the region exterior to $\Omega$, continuity of $\boldsymbol{H}$ across the boundary $\partial \Omega$, and the usual nucleation condition on $\boldsymbol{\omega}$. However, now note that if $J_{n u c l}<J_{c \perp}$ then even though vortices are nucleated at the boundary when $|\boldsymbol{J}|=J_{\text {nucl }}$ they will not be transported into the sample until $|\boldsymbol{J}|=J_{c \perp}$, so that $J_{\text {nucl }}$ is redundant in this case.

\subsection{Two dimensions}

As usual, in two dimensions there are no problems of instability, and we therefore have only one critical current. We find

$$
\begin{aligned}
-\operatorname{div}\left(\frac{1}{\hat{a}(\boldsymbol{x})} \nabla H\right)+H & =\omega, \\
\omega_{t}+\operatorname{div}(\omega \boldsymbol{v}) & =0, \\
\boldsymbol{v}=-\operatorname{sign}(\omega) \bar{F}(|\nabla H| ; \boldsymbol{x}, \boldsymbol{\omega}) \nabla H, &
\end{aligned}
$$

where $\bar{F}(|\nabla H| ; \boldsymbol{x}, \boldsymbol{\omega})=F(|\nabla H| ; \boldsymbol{x}, \boldsymbol{\omega}) /|\nabla H|$. This model (with $\hat{a} \equiv 1$ ) has been studied recently in $[34,35]$. In particular, in one dimension the existence of a weak solution is proved and the long-time behaviour studied, while numerical simulations are performed in both one and two dimensions. 


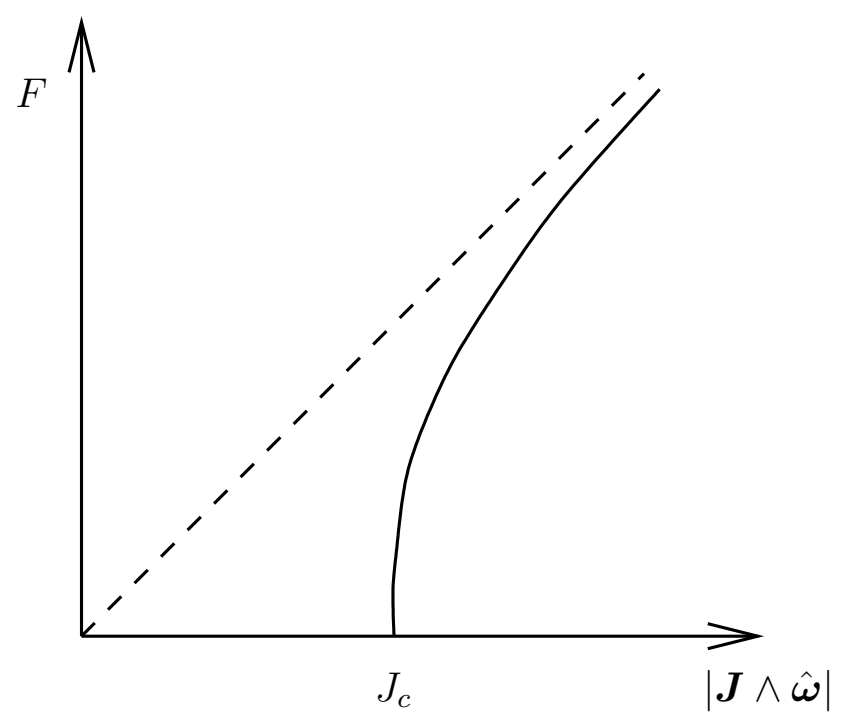

Figure 11: Velocity law with stick-slip pinning.

\subsection{The long-wave limit}

In the long-wave limit the stick-slip pinning model becomes

$$
\begin{aligned}
\boldsymbol{H} & =\boldsymbol{\omega}, \\
\frac{\partial \boldsymbol{H}}{\partial t}+\operatorname{curl} \boldsymbol{E} & =0, \\
\boldsymbol{E} & =\boldsymbol{H} \wedge \boldsymbol{v}+G(|\boldsymbol{J} \cdot \hat{\boldsymbol{H}}|,|\boldsymbol{J}|)|\boldsymbol{H}| \hat{\boldsymbol{J}}, \\
\boldsymbol{v} & =F(|\boldsymbol{J} \wedge \hat{\boldsymbol{H}}|) \hat{\boldsymbol{J}} \wedge \hat{\boldsymbol{H}},
\end{aligned}
$$

where we have not indicated explicitly the dependence of $F$ and $G$ on $\boldsymbol{x}$ and $\boldsymbol{\omega}$. In two dimensions this becomes

$$
H_{t}=\operatorname{div}(|H| \bar{F}(|\nabla H|) \nabla H) .
$$

\subsection{The infinite mobility limit}

The models above are critical-state models, since the vortices do not move until the current density exceeds a critical value (in fact, the term critical state is used to refer to the fact that the solution of these models will have regions in which the current density is exactly at the critical value, since this is the point at which vortices stop moving). In the models described thus far the vortices have a finite mobility once the current exceeds the critical value.

There is a further asymptotic limit which we may take, which reduces these models to better known critical-state models. If we assume that the timescale for changes in the applied magnetic field is small by comparison to the timescale for vortex motion above $J_{c}$ then we may replace the graph shown in Figure 11 by that shown in Figure 12. This means that the vortices effectively have infinite mobility for current densities above $J_{c}$, and will rearrange themselves instantaneously on 


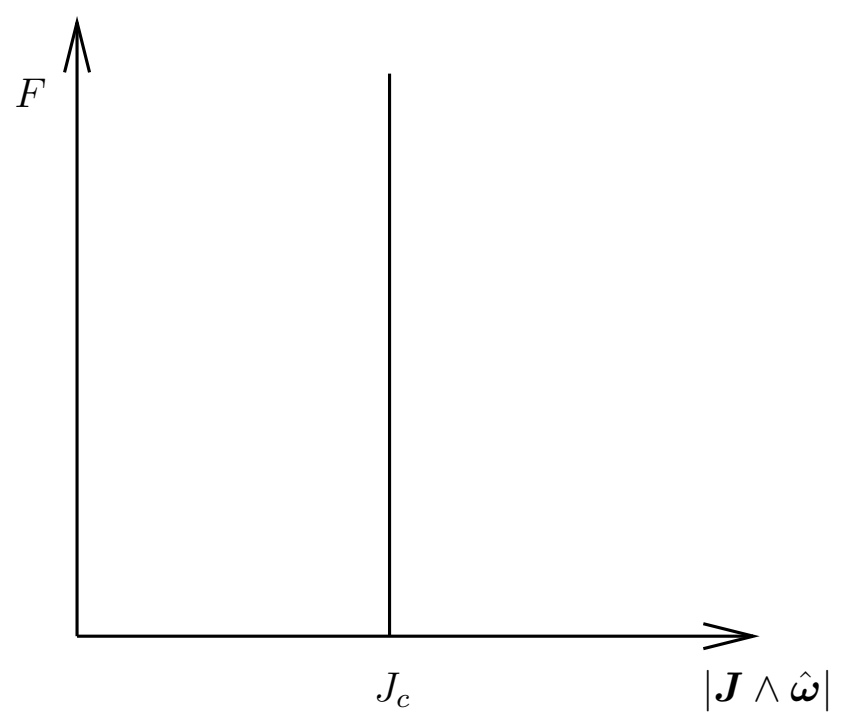

Figure 12: Velocity law in the critical state model.

the timescale of variations in the applied magnetic field. These models have the advantage of being independent of the details of vortex motion above $J_{c}$, so that we do not need to know the forms of the functions $F$ and $G$. We will now describe them, assuming for simplicity that $\hat{a}=1$.

We consider first the case in which $\boldsymbol{\omega} \cdot \boldsymbol{J} \equiv 0$. The velocity of the vortices is now given by a graph of $|\boldsymbol{J} \wedge \hat{\boldsymbol{\omega}}|=|\boldsymbol{J}|$ rather than a function of $|\boldsymbol{J}|$ : the velocity is unknown when $|\boldsymbol{J}|>J_{c}$, and is determined by the constraint that $|\boldsymbol{J}|$ can never be greater than $J_{c}$. The model becomes

$$
\begin{aligned}
(\operatorname{curl})^{2} \boldsymbol{H}+\boldsymbol{H} & =\boldsymbol{\omega}, \\
\operatorname{div} \boldsymbol{H} & =0, \\
\frac{\partial \boldsymbol{\omega}}{\partial t}+\operatorname{curl}(\boldsymbol{\omega} \wedge \boldsymbol{v}) & =0, \\
\boldsymbol{v} & =m \hat{\boldsymbol{J}} \wedge \hat{\boldsymbol{\omega}}
\end{aligned}
$$

where $\hat{\boldsymbol{J}}$ is the unit vector in the direction of $\boldsymbol{J}$, and $m$ is to be determined as part of the problem. To these equations we must add the constraints

$$
\begin{aligned}
m & \geq 0 \\
|\boldsymbol{J}| & \leq J_{c} \quad \text { if }|\boldsymbol{\omega}| \neq 0 \\
m & =0 \quad \text { if }|\boldsymbol{J}|<J_{c} .
\end{aligned}
$$

Thus either the vortices are stationary and the current density is less than $J_{c}$, or the current density is equal to $J_{c}$ and we know the direction but not the magnitude of the vortex motion. To these equations we add as usual the Maxwell equations (63) in the region outside $\Omega$, along with continuity of $\boldsymbol{H}$ across $\partial \Omega$ and a condition at infinity. However, we still need to give a boundary condition on $\boldsymbol{\omega}$ to determine the nucleation of vorticity at the boundary, since (166) holds only if $|\boldsymbol{\omega}| \neq 0$. At present with an initially empty sample the solution $\boldsymbol{\omega} \equiv \mathbf{0}$ is good for all applied magnetic fields. 
In fact vortices will be generated whenever $|\boldsymbol{J}|>J_{\text {nucl }}$ on the boundary, but as we have seen, will not move if $J_{\text {nucl }}<J_{c}$ until $|\boldsymbol{J}|=J_{c}$ on the boundary ${ }^{6}$. Hence the generation of vortices at the boundary is controlled by the constraint

$$
|\boldsymbol{J}| \leq J_{c} \quad \text { on } \partial \Omega
$$

also. Now, in the case $\boldsymbol{\omega} \cdot \boldsymbol{J}=0$, there are various simplifications which can be made. Since

$$
\boldsymbol{\omega} \wedge(\hat{\boldsymbol{J}} \wedge \hat{\boldsymbol{\omega}})=|\boldsymbol{\omega}| \hat{\boldsymbol{J}}
$$

(163) may be written

$$
\frac{\partial \boldsymbol{\omega}}{\partial t}+\operatorname{curl}(m|\boldsymbol{\omega}| \hat{\boldsymbol{J}})=\mathbf{0}
$$

Now, we would like to replace $m|\boldsymbol{\omega}|$ in (169) by $m$, by absorbing $|\boldsymbol{\omega}|$ into $m$, but to do so we need to show that (166), (167) hold also when $|\boldsymbol{\omega}|=0$. However, it follows from the maximum principle applied to the curl of (161) that the maximum of $|\boldsymbol{J}|$ over any vortex-free region occurs on the boundary of that region. Since $|\boldsymbol{J}| \leq J_{c}$ on $\partial \Omega$ and in vortex regions it follows that $|\boldsymbol{J}|<J_{c}$ in vortex-free regions, and the model becomes

$$
\begin{aligned}
\frac{\partial \boldsymbol{\omega}}{\partial t}+\operatorname{curl}(m \boldsymbol{J}) & =\mathbf{0}, \\
\operatorname{div} \boldsymbol{H} & =0, \\
\operatorname{curl}^{2} \boldsymbol{H}+\boldsymbol{H} & =\boldsymbol{\omega}, \\
m & \geq 0, \\
|\boldsymbol{J}| & \leq J_{c} \\
m & =0 \quad \text { if }|\boldsymbol{J}|<J_{c} .
\end{aligned}
$$

Note that

$$
\boldsymbol{E}=\boldsymbol{J}_{t}+m \boldsymbol{J}
$$

so that the model is very similar to that proposed in Section 5 , although $m$ is now determined by the constraint on the magnitude of $\boldsymbol{J}$, rather than a constraint on the helicity of $\boldsymbol{H}$.

Let us now move on to consider the fully three-dimensional problem in which $\boldsymbol{\omega} \cdot \boldsymbol{J} \not \equiv 0$ and we must model the effects of the helical instability. Including these as before gives

$$
\begin{aligned}
(\operatorname{curl})^{2} \boldsymbol{H}+\boldsymbol{H} & =\boldsymbol{\omega} \\
\operatorname{div} \boldsymbol{H} & =0 \\
\frac{\partial \boldsymbol{H}}{\partial t}+\operatorname{curl} \boldsymbol{E} & =\mathbf{0} \\
\boldsymbol{E} & =\boldsymbol{J}_{t}+\boldsymbol{\omega} \wedge \boldsymbol{v}+m_{\|} \boldsymbol{J}, \\
\boldsymbol{v} & =m_{\perp} \hat{\boldsymbol{J}} \wedge \hat{\boldsymbol{\omega}},
\end{aligned}
$$

with the constraints

$$
\begin{aligned}
m_{\perp} \geq 0, & m_{\|} \geq 0, \\
|\boldsymbol{J} \wedge \hat{\boldsymbol{\omega}}| \leq J_{c \perp}, & |\boldsymbol{J} \cdot \hat{\boldsymbol{\omega}}| \leq J_{c \|} \quad \text { if }|\boldsymbol{\omega}| \neq 0, \\
m_{\perp}\left(|\boldsymbol{J} \wedge \hat{\boldsymbol{\omega}}|-J_{c \perp}\right)=0, & m_{\|}\left(|\boldsymbol{J} \cdot \hat{\boldsymbol{\omega}}|-J_{c \|}\right)=0,
\end{aligned}
$$

\footnotetext{
${ }^{6}$ If $J_{\text {nucl }}>J_{c}$ there is a rapid transient just after vortex nucleation, since the constraint (166) is suddenly not satisfied. To describe the solution during this transient we must return to the previous model with finite vortex mobility. We therefore consider here only the case $J_{n u c l}<J_{c}$.
} 
with Maxwell's equations outside $\Omega$ as usual, continuity of $\boldsymbol{H}$ across $\partial \Omega$, and the constraints

$$
\begin{aligned}
|\boldsymbol{J}| & \leq J_{c \perp} \quad \text { on } \partial \Omega, \\
|\boldsymbol{J} \cdot \hat{\boldsymbol{\omega}}| & =0 \quad \text { if }|\boldsymbol{J}|=J_{c \perp} \quad \text { on } \partial \Omega,
\end{aligned}
$$

the latter constraint saying that new vortices are generated perpendicular to $\boldsymbol{J}$.

Two dimensions A particular case in which $\boldsymbol{J} \cdot \hat{\boldsymbol{\omega}} \equiv 0$ is our usual two-dimensional setting. In this case the model simplifies substantially to become

$$
\begin{aligned}
\frac{\partial \omega}{\partial t} & =\operatorname{div}(m \nabla H), \\
-\nabla^{2} H+H & =\omega,
\end{aligned}
$$

with the constraints

$$
m \geq 0, \quad|\nabla H| \leq J_{c}, \quad m\left(J_{c}-|\nabla H|\right)=0 .
$$

This model has been studied analytically and numerically recently in [28]. There is an equivalent variational formulation [54], which is to find $H \in K$ such that

$$
\begin{aligned}
\left(\omega_{t}, \phi-H\right) & \geq 0, \quad \forall \phi \in K, \\
\left.\omega\right|_{t=0} & =\omega_{0}, \\
\omega & =H-\nabla^{2} H,
\end{aligned}
$$

where

$$
K=\left\{\phi \text { such that }|\nabla \phi| \leq J_{c},\left.\phi\right|_{\partial D}=H_{e x t}\right\}
$$

and

$$
(f, g)=\int_{D} f g d x .
$$

If $J_{c}$ is independent of $H$ then this is a variational inequality; if $J_{c}$ depends on $H$ it is quasivariational, since the set $K$ of admissible functions then depends on the solution.

For the case of a virgin sample in an increasing magnetic field there will be a region around the perimeter in which $|\nabla H|=J_{c}$ and a region in the interior in which $\omega=0$.

\subsection{Infinite mobility in the long-wave limit}

As with the vortex-density models, we can consider the limit in which the size of the sample, $L_{D}$, is much greater than the penetration depth, $\lambda_{D}$. Rescaling lengths with $L_{D} / \lambda_{D}$ and time with $L_{D}^{2} / \lambda_{D}^{2}$ as usual, and letting $\lambda=\lambda_{D} / L_{D} \rightarrow 0$, we find

$$
\begin{aligned}
\boldsymbol{H} & =\boldsymbol{\omega}, \\
\operatorname{div} \boldsymbol{H} & =0, \\
\frac{\partial \boldsymbol{H}}{\partial t}+\operatorname{curl} \boldsymbol{E} & =\mathbf{0}, \\
\boldsymbol{E} & =\hat{\boldsymbol{H}} \wedge \boldsymbol{v}+m_{\|} \boldsymbol{J}, \\
\boldsymbol{v} & =m_{\perp} \boldsymbol{J} \wedge \hat{\boldsymbol{H}},
\end{aligned}
$$


with the constraints that

$$
\begin{aligned}
m_{\perp} \geq 0, & m_{\|} \geq 0, \\
|\boldsymbol{J} \wedge \hat{\boldsymbol{H}}| \leq J_{c \perp}, & |\boldsymbol{J} \cdot \hat{\boldsymbol{H}}| \leq J_{c \|}, \\
m_{\perp}\left(|\boldsymbol{J} \wedge \hat{\boldsymbol{H}}|-J_{c \perp}\right)=0, & m_{\|}\left(|\boldsymbol{J} \cdot \hat{\boldsymbol{H}}|-J_{c \|}\right)=0 .
\end{aligned}
$$

As usual, these equations are coupled to the Maxwell equations (63) outside $\Omega$, with continuity of $\boldsymbol{H}$ across $\partial \Omega$ and $\boldsymbol{H} \rightarrow \boldsymbol{H}_{\text {ext }}$ at infinity.

The model (198)-(202) is very similar to the "double critical state" model written down by Clem \& Perez-Gonzales [25]. They considered a one-dimensional situation in which all fields were functions of $x$ and $t$ only, and postulated that there should be "parallel" and "perpendicular" critical currents as in (201). However, they postulated that the electric field should be given by

$$
\boldsymbol{E}=m_{\perp} \boldsymbol{J}_{\perp}+m_{\|} \boldsymbol{J}_{\|},
$$

where $\boldsymbol{J}_{\perp}$ and $\boldsymbol{J}_{\|}$are the components of $\boldsymbol{J}$ perpendicular and parallel to $\boldsymbol{H}$ respectively, in contrast to (198), which may be written

$$
\boldsymbol{E}=m_{\perp} \boldsymbol{J}_{\perp}+m_{\|} \boldsymbol{J}
$$

Mathematically, (203) is the more natural equation, since in that case $\boldsymbol{E}$ is a subdifferential of the characteristic function of the admissible set specified by the constraint, i.e., of a function equal to zero inside and to infinity outside the set. If we wish this to be true with $\boldsymbol{E}$ given by (204) the constraint should be on $|\boldsymbol{J}|$ rather than on $\left|\boldsymbol{J}_{\|}\right|$. However, the two terms generating the electric field in (204) involve quite different mechanisms on the vortex scale. The first is a sort of "dry friction", so that the vortices move in the direction of the force on them once this force exceeds a critical value. The second is generated by the helical instability, which is initiated when $\boldsymbol{J}_{\|}$becomes too large, but the evolution of which is governed predominantly by $\boldsymbol{J}$. However, we have not made at all clear how the pinning forces affect the nucleation and evolution of the helical instability, so that even though we have tried to take account of what is happening on the small scale in writing down (204), it is not clear that we have ended with a sensible mathematical problem.

We note that it is difficult to choose between the equations (203) and (204) on the basis of available experimental evidence, partly because experimental arrangements are often chosen which exhibit a symmetry which leads to $\boldsymbol{J} \cdot \boldsymbol{H} \equiv 0$, and partly because neither model has been studied in any detail mathematically or simulated numerically.

As usual, the complications above disappear if symmetry implies that $\boldsymbol{J} \cdot \boldsymbol{H} \equiv 0$. In this simpler case (198)-(199) may be written

$$
\boldsymbol{E}=m \boldsymbol{J}
$$

with the constraints

$$
m \geq 0, \quad|\boldsymbol{J}| \leq J_{c \perp}, \quad m\left(|\boldsymbol{J}|-J_{c \perp}\right)=0 .
$$

This model and its equivalent variational formulations have been studied extensively by Prigozhin $[51,53,52]$.

Two dimensions In our usual two-dimensional setting the model again simplifies greatly. We find

with the constraints

$$
\frac{\partial H}{\partial t}=\operatorname{div}(m \nabla H)
$$

$$
m \geq 0, \quad|\nabla H| \leq J_{c}, \quad m\left(J_{c}-|\nabla H|\right)=0 .
$$


As usual, in this case we have the Dirichlet condition $H=H_{\text {ext }}$ on $\partial D$. This is the most commonly studied critical state model. When $J_{c}$ is constant it is known as the Bean model [7, 8], and when $J_{c}$ is a function of $H$ it is known as the Kim-Anderson model [43, 44, 2].

As in the finite $\lambda$ case, there is an equivalent variational formulation [51], which is to find $H \in K$ such that

$$
\begin{aligned}
\left(H_{t}, \phi-H\right) & \geq 0, \quad \forall \phi \in K, \\
\left.H\right|_{t=0} & =H_{0},
\end{aligned}
$$

where $K$ is as in (193). As before, the inequality is quasi-variational if $J_{c}$ depends on $H$, and variational if not. For the case of a virgin sample in an increasing magnetic field there will be a region around the outside in which $|\nabla H|=J_{c}$ and a region in the interior in which $H=0$ and no vortices are present.

\section{Conclusion}

We have aimed to show how the mesoscopic Ginzburg-Landau model for superconductivity, which is well established experimentally and popular for study by both mathematicians and physicists, is related to the macroscopic critical-state models popular among engineers. The relationship comes from taking a series of asymptotic limits which generate a hierarchy of models each with a different range of validity, as illustrated in Figure 13. In any given experimental or practical situation the parameters can be evaluated to decide which model is the most appropriate, depending also on the accuracy required in the solution. The mathematical analysis of many of the models we have presented is in its infancy, although some results are beginning to appear.

We have presented both the commonly-studied and much simpler two-dimensional model for a cylinder in an axial magnetic field, as well as the general three-dimensional model, which must at each stage be coupled to Maxwell's equations in the region exterior to the superconducting material and so poses new problems over and above the obvious complexity of the third dimension and a multi-directional magnetic field. The three-dimensional model also has the possibility of a small-scale instability of vortex filaments in situations with no special symmetry which reverberates throughout the hierarchy leading to extra complications in the fully general problem.

An interesting feature of the validity of the vortex-density models is the determination of the boundary between regions where local forces dominate the electromagnetic balance and those where mean-field forces dominate, which corresponds to the switch from a vortex lattice to a vortex liquid. This transition is usually thought of as being controlled by the temperature of the material in a manner analogous to the usual melting of a crystalline solid. However, under the isothermal conditions we have considered this switch depends on the relative scalings of the magnetic field and the electric current, as illustrated in Figure 5. If the magnetic field varies over a lengthscale comparable to the sample size (as we might expect), then this limits the vortex liquid models to samples of size $L_{D} \ll \kappa \lambda_{D}$. When local forces dominate the bulk motion of vortices will occur through dislocations in the vortex lattice, which is a problem analogous to finding a macroscopic model of two-dimensional plastic flow based on local models of elastic crystal lattices. The dependence of the bulk properties of the solution on the details of the local behaviour makes such a situation more difficult to model, and poses an interesting open problem.

Acknowledgements The author would like to acknowledge the help of Dr Malcolm McCulloch, Prof David Dew-Hughes and Dr Gary Barnes in providing photographs and numerical simulations of superconducting motors. 


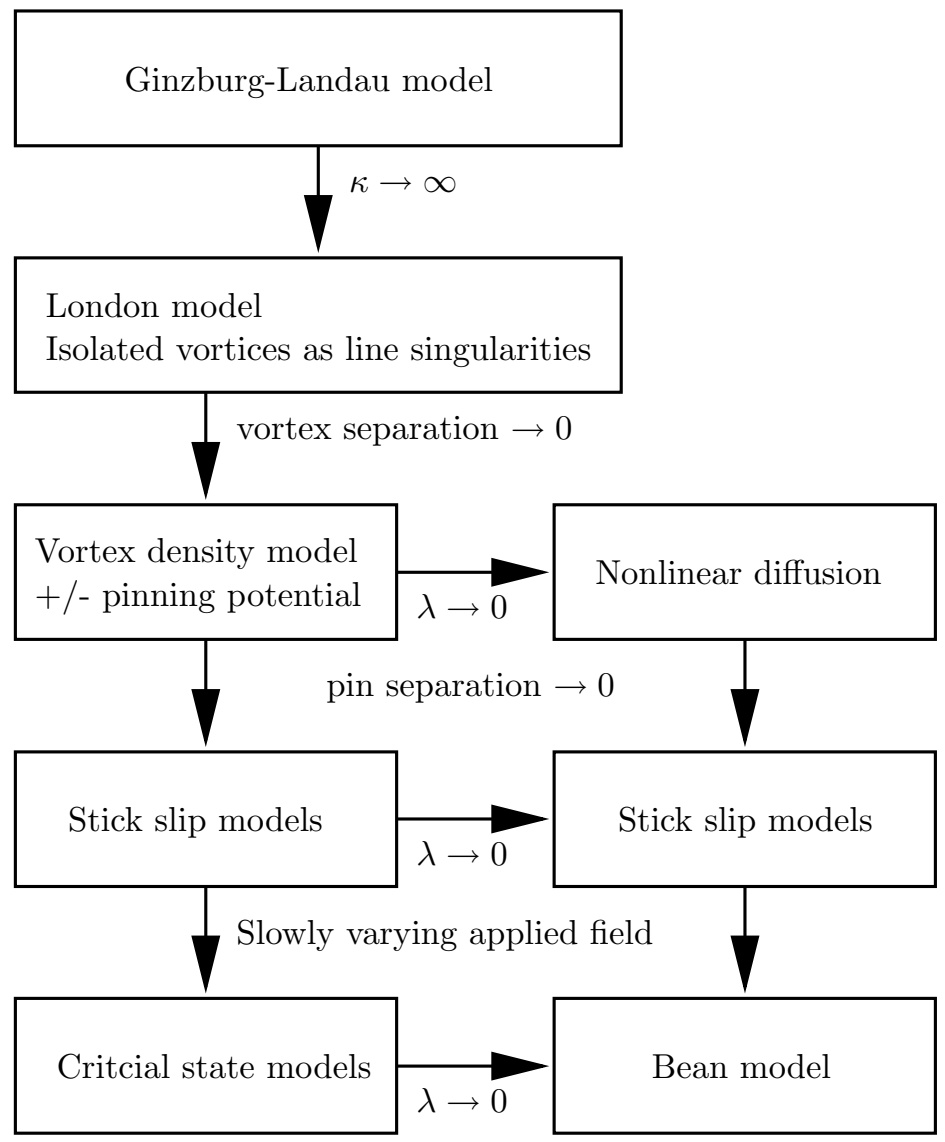

Figure 13: The hierarchy of models for type II superconductors. 


\section{References}

[1] A. A. Abrikosov. On the magnetic properties of superconductors of the second group. Soviet Phys. JETP, 5(6):1174-1182, 1957.

[2] P. W. Anderson. Theory of flux creep in hard superconductors. Phys. Rev. Lett., 9:309-311, 1962.

[3] D. G. Aronson. The porous medium equation. Lecture notes in Mathematics 1224, Springer Verlag, pages 1-46, 1986.

[4] J. Bardeen, L. N. Cooper, and J. R. Schrieffer. Theory of superconductivity. Phys. Rev., 108:1175-1204, 1957.

[5] G. J. Barnes, M. D. McCulloch, and D. Dew-Hughes. Computer modelling of type ii superconductors in applications. Supercond. Sci. Technol., 12(8):518-522, 1999.

[6] G. J. Barnes, M. D. McCulloch, and D. Dew-Hughes. Finite difference modelling of bulk high temperature superconducting cylindrical hysteresis machines. Supercond. Sci. Technol., 13:229-236, 2000.

[7] C. P. Bean. Magnetization of hard superconductors. Phys. Rev. Lett., 8:250-253, 1962.

[8] C. P. Bean. Magnetization of high-field superconductors. Reviews of Modern Physics, 36:31-39, 1964.

[9] M. S. Berger and Y. Y. Chen. Symmetric vortices for the Ginzburg-Landau equations of superconductivity and the nonlinear desingularization phenomenon. J. Funct. Anal., 82:259$295,1989$.

[10] A. Bonnet, S. J. Chapman, and R. Monneau. Convergence of Meissner minimisers of the Ginzburg-Landau energy of superconductivity as $\kappa \rightarrow+\infty$. SIAM J. Math. Anal., to appear, 2000 .

[11] A. Bonnet and R. Monneau. Distribution of vortices in a type-II superconductor as a free boundary problem: Existence and regularity via Nash-Moser theory. Interfaces and Free Boundaries, 2(2):181-200, 2000.

[12] E. H. Brandt. Flux diffusion in high- $T_{c}$ superconductors. Z. Phys. B, 80:167-175, 1990.

[13] S. J. Chapman. A mean-field model of superconducting vortices in three dimensions. SIAM J. Appl. Math., 55(5):1259-1274, 1995.

[14] S. J. Chapman. The superheating field of type-II superconductors. SIAM J. Appl. Math., 55(5):1233-1258, 1995.

[15] S. J. Chapman, Q. Du, and M. D. Gunzburger. A Ginzburg-Landau-type model of superconducting/normal junctions including Josephson junctions. Europ. J. Appl. Math., 6:97-114, 1995.

[16] S. J. Chapman and D. R. Heron. The motion of superconducting vortices in thin films of varying thickness. SIAM J. Appl. Math., 58(6):1808-1825, 1998. 
[17] S. J. Chapman, S. D. Howison, and J. R. Ockendon. Macroscopic models of superconductivity. SIAM Review, 34(4):529-560, 1992.

[18] S. J. Chapman and G. Richardson. Motion of vortices in type-II superconductors. SIAM J. Appl. Math., 55(5):1275-1296, 1995.

[19] S. J. Chapman and G. Richardson. Vortex pinning by inhomogeneities in type-II superconductors. Physica D, 108(4):397-407, 1997.

[20] S. J. Chapman and G. Richardson. Personal communication. 1999.

[21] S. J. Chapman, J. Rubinstein, and M. Schatzman. A mean-field model of superconducting vortices. Europ. J. Appl. Math., 7:97-111, 1996.

[22] X. Chen, C. M. Elliott, and Q. Tang. Shooting method for vortex solutions of a complex-valued Ginzburg-Landau equation. Proc. Roy. Soc. Edin. A, 124(6):1075-1088, 1994.

[23] Z. Chen and K. H. Hoffmann. Numerical simulations of dynamical Ginzburg-Landau vortices in superconductivity. In M. Griebel and C. Zenger, editors, Proc. of the FORTWIHR Symposium on High Performance Scientific Computing, München, June 17-18 1993, pages 31-38. Braunschweig:Vieweg, 1994.

[24] J. R. Clem. Spiral vortex interaction instability in type-II superconductors. Phys. Rev. Lett., $38: 1425-1428,1977$.

[25] J. R. Clem and A. Perez-Gonzales. Theory of the double critical state in type-II superconductors. In U. Eckern, A. Schmid, W. Weber, and H. Wühl, editors, Proc. of LT-17, pages 583-584. Elsevier, 1984.

[26] J. Deang, Q. Du, M. D. Gunzburger, and J. Peterson. Vortices in superconductors: Modelling and computer simulations. Phil. Trans. A, 355(1731):1957-1968, 1997.

[27] A. T. Dorsey. Vortex motion and the Hall effect in type-II superconductors: A time-dependent Ginzburg-Landau theory approach. Phys. Rev. B, 46(13):8376-8392, 1992.

[28] Q. Du, M. D. Gunzburger, and H. K. Lee. Analysis and computation of a mean-field model for superconductivity. Num. Math., 81(4):539-560, 1999.

[29] Q. Du, M. D. Gunzburger, and J. Peterson. Solving the Ginzburg-Landau equations by finiteelement methods. Phys. Rev. B, 46(14):9027-9034, 1992.

[30] Q. Du, M. D. Gunzburger, and J. Peterson. Modeling and analysis of a periodic GinzburgLandau model for type-II superconductors. SIAM J. Appl. Math., 53(3):689-717, 1993.

[31] Q. Du, M. D. Gunzburger, and J. S. Peterson. Analysis and approximation of the GinzburgLandau model of superconductivity. SIAM Review, 34(1):54-81, 1992.

[32] W. E. Dynamics of vortices in Ginzburg-Landau theories with applications to superconductivity. Physica D, 77(4):383-404, 1994.

[33] C. M. Elliott, R. Schätzle, and B. Stoth. Viscosity solutions of a degenerate parabolic elliptic system arising in the mean field theory of superconductivity. Arch. Rat. Mech. Anal., 145(2):99-127, 1998. 
[34] C. M. Elliott and V. Styles. Flux pinning and boundary nucleation of vorticity in a mean field model of superconducting vortices. Interfaces and Free Boundaries, 2(2):143-180, 2000.

[35] C. M. Elliott and V. Styles. Numerical analysis of a mean field model of superconducting vortices. IMA J. Num. Anal., to appear, 2000.

[36] V. L. Ginzburg and L. D. Landau. On the theory of superconductivity. JETP, 20:1064-1082, 1950.

[37] L. P. Gor'kov. Microscopic derivation of the Ginzburg-Landau equations in the theory of superconductivity. Soviet Phys. JETP, 9:1364-1367, 1959.

[38] L. P. Gor'kov and G. M. Éliashberg. Generalisation of the Ginzburg-Landau equations for nonstationary problems in the case of alloys with paramagnetic impurities. Soviet Phys. JETP, $27: 328-334,1968$.

[39] L. P. Gor'kov and N. B. Kopnin. Viscous vortex flow in superconductors with paramagnetic impurities. Soviet Phys. JETP, 33:1251-1256, 1971.

[40] W. D. Gropp, H. G. Kaper, G. K. Leaf, D. M. Levine, M. Palumbo, and V. M. Vinokur. Numerical simulation of vortex dynamics in type-II superconductors. J. Comp. Phys., 123(2):254-266, 1996.

[41] H. G. Kaper. Mathematical models of superconductivity. Technical Report ANL/MCS-TM146, Mathematics and Computer Science Division, Argonne National Laboratory, 1991.

[42] R. Kato, Y. Enomoto, and S. Maekawa. Effects of the surface boundary on the magnetization process in type-II superconductors. Phys. Rev. B, 47(13):8016-8024, 1993.

[43] Y. B. Kim, C. F. Hempstead, and A. R. Strnad. Critical persistent currents in hard superconductors. Phys. Rev. Lett., 9:306-309, 1962.

[44] Y. B. Kim, C. F. Hempstead, and A. R. Strnad. Magnetization and critical currents. Phys. Rev., 129:528-535, 1963.

[45] K. Likharev. Superconducting weak links. Rev. Modern Phys., 51:101-159, 1979.

[46] F. London. Superfluids. Dover, 1961.

[47] F. London and H. London. The electromagnetic equations of the supraconductor. Proc. Roy. Soc. Lond. A, 14(9):71, 1935.

[48] M. C. Marchetti and D. R. Nelson. Hydrodynamics of flux liquids. Phys. Rev. B, 42(16):99389943, 1990.

[49] J. C. Neu. Vortices in complex scalar fields. Physica D, 43(2-3):385-406, 1990.

[50] L. Peres and J. Rubinstein. Vortex dynamics in $U(1)$ Ginzburg-Landau models. Physica D, 64:299-309, 1993.

[51] L. Prigozhin. The Bean model in superconductivity: Variational formulation and numerical solution. J. Comp. Phys., 129(1):190-200, 1996. 
[52] L. Prigozhin. On the Bean critical-state model in superconductivity. Europ. J. Appl. Math., 7:237-248, 1996.

[53] L. Prigozhin. Analysis of critical-state problems in type-II superconductivity. IEEE Trans. Appl. Super., 7(4):3866-3873, 1997.

[54] L. Prigozhin. Personal communication. 1998.

[55] G. Richardson. Instability of a superconducting line vortex. Physica D, 110(1-2):139-153, 1997.

[56] G. Richardson and B. Stoth. Ill-posedness of the mean-field model of superconducting vortices and a possible regularisation. Europ. J. Appl. Math., 11(2):137-152, 2000.

[57] G. W. Richardson. Vortex Motion in Type-II Superconductors. PhD thesis, Oxford University, 1995.

[58] E. Sandier and S. Serfaty. Global minimizers for the Ginzburg-Landau functional below the first critical magnetic field. Annales de l'institut Henri Poincare-Analyse Non Lineaire, 17(1):119145,2000 .

[59] E. Sandier and S. Serfaty. On the energy of type-II superconductors in the mixed phase. Preprint, 2000.

[60] E. Sandier and S. Serfaty. A rigorous derivation of a free-boundary problem arising in superconductivity. Preprint, 2000.

[61] R. Schätzle and B. Stoth. The stationary mean field model of superconductivity: Partial regularity of the free boundary. J. Diff. Eqn., 157(2):319-328, 1999.

[62] R. Schätzle and V. Styles. Analysis of a mean field model of superconducting vortices. Europ. J. Appl. Math., 10(4):319-352, 1999.

[63] A. Schmid. A time dependent Ginzburg-Landau equation and its application to the problem of resistivity in the mixed state. Physik der Kondensierten Materie, 5:302, 1966.

[64] V. Styles. The Dynamics of Superconducting Vortices. PhD thesis, University of Sussex, 1996. 